\title{
Schülerhandlungen als Elemente fachdidaktisch motivierter Kompetenzmodellierungen
}

\author{
Renate Nitsch $^{1} \cdot$ Regina Bruder $^{1}$ Augustin Kelava ${ }^{2}$
}

Eingegangen: 23. März 2013 / Angenommen: 6. Dezember 2015 / Online publiziert: 16. Februar 2016 (C) Die Autor(en) 2016. Dieser Artikel ist auf Springerlink.com mit Open Access verfügbar.

Zusammenfassung Als ein möglicher Weg zur Weiterentwicklung des Mathematikunterrichts wird derzeit eine Modellierung der angestrebten fachspezifischen und übergreifenden Kompetenzen der Lernenden angesehen. Etwaige Kompetenzmodellierungen in diesen Bereichen benötigen einen soliden theoretischen Hintergrund $u$. a. auch zur validen Operationalisierung von Kompetenzfacetten in Form von Testitems. In der hier berichteten Studie wurde im Inhaltsbereich der Darstellungswechsel funktionaler Zusammenhänge (Projekt HEUREKO) eine Kompetenzstruktur theoretisch hergeleitet und empirisch überprüft, in dem die für diesen Bereich relevanten grundlegenden mathematischen Schülerhandlungen modelliert wurden. Mithilfe eines im Projekt HEUREKO erhobenen Datensatzes konnte in einer (Re-)Analyse gezeigt werden, dass sich Handlungen des „Identifizierens" und „Realisierens“ mathematischer Inhalte in ihrem Anforderungsprofil unterscheiden, d. h. dass ihnen verschiedene Kompetenzfacetten zugrunde liegen. Diese sind wiederum von Handlungen des „Beschreibens und Begründens“ abgrenzbar. In einem Ausblick werden die Potenziale des Ansatzes der Schülerhandlungen zur Itemkonstruktion und Beschreibung von Kompetenzfacetten für künftige Kompetenzmodellierungen diskutiert.

Renate Nitsch

nitsch@mathematik.tu-darmstadt.de

Regina Bruder

bruder@mathematik.tu-darmstadt.de

Augustin Kelava

augustin.kelava@uni-tuebingen.de

1 Fachbereich Mathematik, Technische Universität Darmstadt, Schlossgartenstraße 7, 64289 Darmstadt, Deutschland

2 Hector-Institut für Empirische Bildungsforschung, Universität Tübingen, Europastraße 6, 72072 Tübingen, Deutschland 
Schlüsselwörter Kompetenzstruktur · Elementarhandlungen · Grundhandlungen · Darstellungswechsel funktionaler Zusammenhänge · Tätigkeitskonzept

MESC D50 $\cdot \mathrm{D} 60 \cdot \mathrm{C} 30 \cdot \mathrm{C} 80 \cdot \mathrm{I} 20$

\title{
Student actions as elements of competence models
}

\begin{abstract}
Modeling of aspired subject-specific and transversal competencies of students is currently seen as a possibility to provide advancement in the field of mathematical teaching. Such a modeling of competencies requires a solid theoretical background to, amongst others, operationalize competence facets in the form of test items in a valid way. In our study, in the context of the project HEUREKO a competence structure model was developed and empirically verified which describes relevant student actions when translating between different forms of representations in the field of functional relationships. Within a reanalysis of the dataset generated in the project HEUREKO we showed that the two actions Identification and Construction differ in their cognitive demands, i.e. they are based on different competence facets. Simultaneously, these actions can be distinguished from the actions Description and Explanation and could therefore be described as different action dimensions. In the given outlook, the potentials of our approach of student actions were discussed concerning the description of competence facets and future modeling of competencies.
\end{abstract}

Keywords Competence structure $\cdot$ Student actions $\cdot$ Translations between functional relationships $\cdot$ Activity theory

\section{Einleitung}

In den letzten Jahren zeigte sich ein wachsendes Interesse der empirischen Bildungsforschung an Kompetenzmodellierungen für den Mathematikunterricht. Die Entwicklung von theoretisch fundierten und empirisch überprüften Kompetenzmodellen wurde zu einem wichtigen Gegenstand fachdidaktischer Diskussion und stellt nach wie vor eine große Herausforderung dar (Biehler und Leuders 2014).

Kompetenzmodelle zur Beschreibung von Kompetenzen Lernender können in drei verschiedene Gruppen unterteilt werden: Kompetenzstufenmodelle, Kompetenzentwicklungsmodelle und Kompetenzstrukturmodelle (Klieme et al. 2008; Koeppen et al. 2008). Kompetenzstufenmodelle beschreiben, welche situationsspezifischen Anforderungen von Individuen auf bestimmten Kompetenzstufen (meist im Sinne von Anforderungsniveaus) bewältigt werden können. Kompetenzstrukturmodelle beschreiben die allgemeine Struktur einer Kompetenz in einem bestimmten Inhaltsbereich und helfen bei der Aufklärung zugrunde liegender Fähigkeiten. Die Strukturelemente einer Kompetenz werden auch als Dimensionen bezeichnet und können in unterschiedlichen Relationen zueinander stehen. Kompetenzentwicklungsmodelle beschreiben Prozessphasen eines Kompetenzerwerbs. 
Bei allen drei Gruppen von Kompetenzmodellen besteht noch ein enormer Forschungs- und Entwicklungsbedarf sowohl hinsichtlich der theoretischen Begründung als auch der empirischen Absicherung solcher Modelle (Schecker und Parchmann 2006). So entstanden die bisherigen Kompetenzmodellierungen häufig mit einer normativen Perspektive. Ein Beispiel hierfür ist das Formulieren und Setzen von Standards, das die Festlegung von Kompetenzstufen zum Ziel hat. Wünschenswert wäre, dass auch Standards auf Basis theoretisch begründeter und empirisch geprüfter Kompetenzmodelle formuliert und gesetzt werden, was zurzeit noch nicht die Regel ist. Einen Versuch in dieser Richtung unternimmt das von Siller et al. (2014) entwickelte Kompetenzstufenmodell O-M-A für die österreichische MATURA in Mathematik. Hier werden Operieren, Modellieren und Argumentieren als für den Mathematikunterricht zentrale und auch in ihrer Struktur bzw. in ihrem Verlauf voneinander unterscheidbare Schüleraktivitäten angenommen.

Der Fokus unseres Beitrages liegt auf einer theoriegeleiteten Entwicklung und empirischen Überprüfung eines Kompetenzstrukturmodells, in dem für den Inhaltsbereich funktionaler Zusammenhänge grundlegende Schüleraktivitäten bezogen auf relevante Darstellungswechsel funktionaler Zusammenhänge modelliert werden. Dazu erfahren diese Schüleraktivitäten entsprechende Präzisierungen in der Begriffshierarchie Tätigkeit - Handlung - Operation, die aus dem Tätigkeitskonzept (auch Tätigkeitstheorie bzw. activity theory genannt) mit den von Lompscher verwendeten Begrifflichkeiten motiviert sind (vgl. Lompscher 1972). Ziel der hier berichteten Studie ist ein Beitrag zu einer theoretisch und empirisch begründeten Strukturaufklärung von Schüleraktivitäten, die eine besondere Bedeutung für Itemkonstruktionen besitzt, um schließlich auch bestimmte Kompetenzfacetten in einem Stufenmodell abzubilden.

Das Tätigkeitskonzept ist ein psychologisches Konstrukt, das den Menschen und seine Entwicklung in Verbindung mit Kultur und Gesellschaft bringt. Dieses Konzept wurde maßgeblich von Vygotskij (u. a. 1978), Leont'ev (u. a. (1977) und Lurija (u.a. 1992) geprägt und im deutschsprachigen Raum insbesondere von Lompscher (1972; Giest und Lompscher 2006) aufgegriffen und weiterentwickelt. Aktuell wird der Tätigkeitstheorie auch in der internationalen Diskussion verstärkt Aufmerksamkeit gewidmet, z. B. in Verbindung mit Konzepten, die den Einsatz digitaler Werkzeuge im Mathematikunterricht beschreiben (vgl. Vandebrouck et al. 2013). Wir verzichten hier aus Umfangsgründen auf eine Darstellung der Grundlagen der Tätigkeitstheorie und beschränken uns auf die für unser Anliegen notwendigen Begriffe und den gewählten konzeptuellen Zugang. Nach Lompscher (1990) ist ein tätigkeitstheoretisches Herangehen an Lernphänomene $u$. a. auch dadurch gekennzeichnet, dass nach den Wechselbeziehungen zwischen Tätigkeits- und Handlungs(sowie Operations-) Ebene gefragt wird. Wir verwenden dafür einen von Bruder und Brückner (1989) operationalisierten Zugang zu einer hierarchischen Beschreibung von Schüleraktivitäten in Form von geistigen Handlungen. Nach diesem Ansatz lassen sich das Identifizieren und das Realisieren von mathematischen Inhalten als Elementarhandlungen beschreiben (siehe auch Kap. 2.2). Zu den komplexer aufgebauten Grundhandlungen gehören nach diesem Ansatz das Erkennen, Beschreiben, Ausführen (Anwenden) und Begründen sowie ein Verknüpfen - jeweils bezogen auf mathematische Begriffe, Zusammenhänge oder Verfahren, die sich aus 
Abb. 1 5-dimensionales Kompetenzstrukturmodell aus HEUREKO

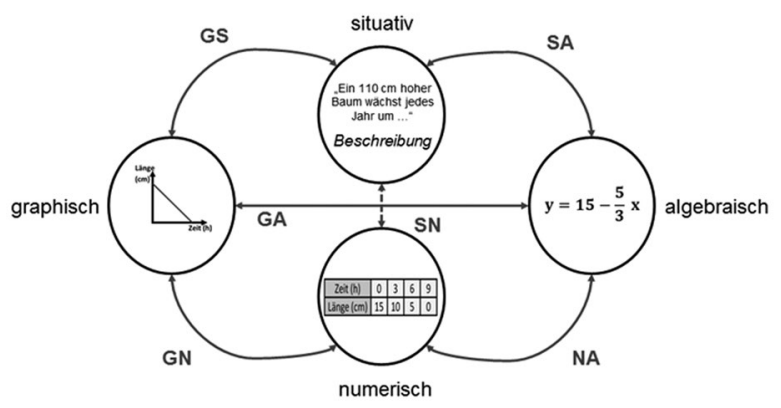

einer rein fachinhaltlichen Betrachtung als zentrale Kategorien für Handlungsgegenstände (Objekte) im Mathematikunterricht ergeben (vgl. Steinhöfel et al. 1985, S. 7).

Dieser theoretische Hintergrund zu relevanten Schüleraktivitäten bei Darstellungswechseln funktionaler Zusammenhänge wurde bereits zur Itemkonstruktion im Rahmen des von der DFG im SPP 1293 „Kompetenzmodelle zur Erfassung individueller Lernergebnisse und zur Bilanzierung von Bildungsprozessen“ geförderten Projektes HEUREKO zusätzlich zur inhaltlichen Fokussierung auf die Darstellungswechsel herangezogen (HEUristisches Arbeiten mit REpräsentationen funktionaler Zusammenhänge und Diagnose mathematischer KOmpetenzen von Schülerinnen und Schülern) und in Expertenratings validiert. Zu den Zielen, Methoden und Ergebnissen des Projektes HEUREKO, das unter Leitung von Leuders, Bruder und Wirtz durchgeführt wurde, sei auf Nitsch et al. (2015) sowie Bayrhuber et al. (2010) verwiesen. Im Folgenden werden die für das Verständnis des Anliegens unserer auf HEUREKO aufbauenden Studie wichtigsten Ergebnisse kurz vorgestellt.

Im Projekt HEUREKO stand die Strukturaufklärung des mathematischen Inhaltsbereiches der Darstellungswechsel funktionaler Zusammenhänge ab Klasse 7 im Mittelpunkt des Forschungsinteresses, da der Umgang mit mathematischen Darstellungsformen und -wechseln als relevanter Bereich mathematischer Allgemeinbildung gilt (Swan 1985; Ainsworth et al. 2002; Vollrath 1989). Der in der zweiten Projektphase in Darmstadt entwickelte Itempool im Projekt HEUREKO für die Klassen 9 und $10 \mathrm{zu}$ linearen und quadratischen Funktionen wurde nach Darstellungswechseln kategorisiert. Hierbei ging es um die Darstellungsformen graphisch, algebraisch, numerisch und situativ. Es wurde ein 5-dimensionales Kompetenzstrukturmodell postuliert, dessen Dimensionen sich aus den unterschiedlichen Darstellungswechseln zusammensetzen (s. Abb. 1):

1. Wechsel zwischen Graph und algebraischer Gleichung (GA)

2. Wechsel zwischen Graph und Wertetabelle (GN)

3. Wechsel zwischen Graph und Situationsbeschreibung (GS)

4. Wechsel zwischen Wertetabelle und algebraischer Gleichung (NA)

5. Wechsel zwischen Situationsbeschreibung und algebraischer Gleichung (SA)

Im Vergleich mit verschiedenen Alternativmodellen konnte das 5-dimensionale Modell empirisch bestätigt werden. Für eine detaillierte Darstellung des Modells und dessen Überprüfung sei auf Nitsch et al. (2015) verwiesen. 
Im vorliegenden Beitrag wird eine Reanalyse des für das Projekt HEUREKO erhobenen Datensatzes berichtet, in der nicht mehr die Darstellungswechsel fokussiert, sondern allein die jeweiligen Schüleraktivitäten in den Blick genommen werden. Es geht also um eine empirische Prüfung eines kognitiven Modells zur Strukturaufklärung von Schüleraktivitäten in einem ersten Anlauf. Mit diesem Vorgehen verbindet sich die Vorstellung, dass die im theoretischen Modell angenommenen Elementarhandlungen (Identifizieren und Realisieren) sowie die komplexeren Grundhandlungen eigenständige und damit auch inhaltsunabhängig zu unterscheidende Konstrukte sind, die sich prinzipiell auch zur Aufklärung von Itemschwierigkeit eignen.

Der Itempool wurde in der Reanalyse also allein unter dem Gesichtspunkt der in den Items angelegten Elementar- und Grundhandlungen betrachtet ohne die Art des jeweiligen Darstellungswechsels zu berücksichtigen, auf die diese Handlungen gerichtet waren. Unsere Hypothese besteht darin, dass sich die theoretisch postulierte Unterscheidbarkeit der beiden Elementarhandlungen Identifizieren und Realisieren unabhängig von den jeweiligen Darstellungswechseln auch tatsächlich empirisch wiederfinden lässt. Darüber hinaus wird erwartet, dass sich die Elementarhandlungen von Grundhandlungen unterscheiden lassen - in unserem Fall geht es um die Grundhandlungen Beschreiben und Begründen, da der Itempool für das Projekt HEUREKO entsprechend konstruiert wurde. Weitergehende Hypothesen lassen sich auf dem vorliegenden Itemsatz nicht bilden, da das Projekt HEUREKO eine andere Zielstellung hatte, so dass zur hier interessierenden Unterscheidung weiterer Schüleraktivitäten und deren möglicher Hierarchie nicht genügend Items vorliegen.

Lässt sich der aufgrund der Definitionen theoretisch plausible Ansatz einer Hierarchie der Schüleraktivitäten in deren kognitiven Anforderungen tatsächlich empirisch verifizieren, wäre unser Ansatz nicht nur für Kompetenzstrukturmodellierungen relevant, sondern könnte auch bei Kompetenzstufenmodellierungen zum Einsatz kommen und wäre für die Konstruktion sowohl von Testitems als auch von Lernaufgaben von besonderer Bedeutung.

Im folgenden zweiten Kapitel wird anhand des zugrunde gelegten Kompetenzbegriffs das Ziel der vorliegenden Studie herausgearbeitet, um dann das gewählte Konstrukt der Elementar- und Grundhandlungen nach Bruder und Brückner (1989) kurz vorzustellen.

Im Zentrum des vorliegenden Beitrages stehen im dritten Kapitel die empirischen Ergebnisse einer Reanalyse des im Projekt HEUREKO generierten Datensatzes zu Darstellungswechseln funktionaler Zusammenhänge, um die Unterscheidbarkeit der theoretisch postulierten Handlungen in einem bereits gut untersuchten Inhaltsbereich zu prüfen. Die Analyse der empirischen Itemschwierigkeiten zeigt erste Hinweise für eine solche Hierarchie,

In einem Ausblick im vierten Kapitel werden die Potenziale des Ansatzes der hierarchisch operationalisierten Schüleraktivitäten in Form von Elementar-und Grundhandlungen zur Beschreibung von Kompetenzfacetten für künftige Kompetenzmodellierungen und Aufgabenentwicklungen für Lehr- und Lernsituationen diskutiert. 


\section{Theoretischer Hintergrund}

\subsection{Zur Operationalisierung des Kompetenzbegriffs}

Die Einführung von Bildungsstandards für den Mathematikunterricht in Verbindung mit dem Weinertschen Kompetenzbegriff hat die fachdidaktische Diskussion um Qualitätsmaßstäbe für den Mathematikunterricht belebt. Weinert (2001) beschreibt Kompetenzen weit und anspruchsvoll als ,,die bei Individuen verfügbaren oder durch sie erlernbaren kognitiven Fähigkeiten und Fertigkeiten, um bestimmte Probleme zu lösen, sowie die damit verbundenen motivationalen, volitionalen und sozialen Bereitschaften und Fähigkeiten, um die Problemlösungen in variablen Situationen erfolgreich und verantwortungsvoll nutzen zu können." (Weinert 2001, S. 27 f.). Dieser Kompetenzbegriff wurde von Weinert in Abwägung unterschiedlicher theoretischer Standpunkte und empirischer Befunde ausgewählt (vgl. Klieme 2004). Weinert betont in diesem Zusammenhang, dass die individuelle Ausprägung von Kompetenz von verschiedenen Facetten bestimmt wird: Fähigkeiten, Wissen, Verstehen, Können, Handeln, Erfahrung, Motivation (vgl. Klieme 2007).

Wir konzentrieren uns im Folgenden auf die Betrachtung kognitiver Aspekte (im Sinne geistiger Handlungen), wohingegen wir motivationale Tendenzen nicht einbeziehen. Diese Einschränkung stützt sich auf den im Projekt HEUREKO wie auch den anderen Projekten in diesem Programm fokussierten, enger gefassten Kompetenzbegriff, indem „Kompetenzen als kontextspezifische kognitive Leistungsdispositionen, die sich funktional auf Situationen und Anforderungen in bestimmten Domänen beziehen“ (Klieme und Leutner 2006, S. 879) betrachtet werden.

Zur Messung von Kompetenzen, bzw. von Kompetenzfacetten, müssen zunächst kognitive Modelle dieser Kompetenzen entwickelt werden, die unterschiedliche Schwerpunktsetzungen haben können (s. Einleitung). Um diese kognitiven Modelle $\mathrm{zu}$ überprüfen, bedarf es geeigneter psychometrischer Modelle. „Psychometric models are the link between theoretical constructs and the results of empirical assessments" (Klieme et al. 2008, S. 12). Geeignet sind hierzu vor allem Modelle der Item-Response Theorie (IRT). Die IRT bietet unter anderem den großen Vorteil, dass Personenfähigkeiten und Itemschwierigkeiten auf einer Skala miteinander verglichen werden können. Zurzeit werden vor allem explanatorische IRT Modelle (Wilson et al. 2008), multidimensionale IRT Modelle und kognitive Diagnosemodelle (Rupp und Mislevy 2007) unterschieden. Unser Ansatz bezieht sich auf ein multidimensionales IRT Modell. Eine übersichtliche Darstellung der Grundlagen multidimensionaler IRT Modelle findet sich z. B. in Hartig und Höhler (2008).

Insgesamt lässt sich feststellen, dass zum jetzigen Zeitpunkt nur wenige empirisch validierte Kompetenzmodelle in der Mathematikdidaktik vorliegen. Häufig müssen die in Frage kommenden psychometrischen Modelle erst auf ihre Eignung für die Kompetenzmodellierung geprüft werden. Dies lässt sich nicht zuletzt darin erkennen, dass im DFG-Schwerpunktprogramm SPP 1293 „Kompetenzmodelle zur Erfassung individueller Lernergebnisse und zur Bilanzierung von Bildungsprozessen“ drei der vier mathematikrelevanten Forschungsprojekte eher psychometrische Modelle und zugehörige Messverfahren in den Vordergrund stellen und diese am Beispiel der Domäne Mathematik realisieren (Heinze 2010, S. 86). Das Projekt HEUREKO ist 
das einzige Projekt, das die Prüfung einer theoretischen Kompetenzstrukturmodellierung bezogen auf mathematische Lerninhalte in den Vordergrund stellt.

\subsection{Ein theoretisches Beschreibungsmodell für Identifizieren, Realisieren, Beschreiben und Begründen}

Für eine Kompetenzmodellierung und deren Operationalisierung in Testaufgaben werden begriffliche Abgrenzungen für relevante Schülerhandlungen benötigt, um beschreiben zu können, was mit den jeweiligen Inhaltselementen „getan“ und im Sinne einer Kompetenzfacette ,gezeigt“" werden soll. Hilfreich für Kompetenzmodellierungen ist ein möglichst präzises, konsensfähiges und konsensbildendes Begriffssystem für relevante Schülerhandlungen. Zumindest die Funktion einer Konsensbildung über Erwartungen in schriftlichen Abiturprüfungen haben die Operatorenlisten für Prüfungsaufgaben im Zentralabitur in mehreren Unterrichtsfächern. ${ }^{1}$ Eine gewisse Präzision der Sprache über Niveaus der Ausprägung von Kompetenzfacetten kann jedoch erst dann erreicht werden, wenn das entsprechende Vokabular gemeinsame begriffliche Wurzeln hat. Im Hinblick auf Kompetenzstufenmodelle sollte es zudem hierarchisch organisiert sein (was bei den Operatoren nicht der Fall ist). Die Tätigkeitstheorie mit den von Kossakowski und Lompscher (1988) vorgestellten Begrifflichkeiten bietet eine (alternative) theoretische Grundlage für Definitionen von aufeinander aufbauenden Schülerhandlungen. Kossakowski und Lompscher forderten für die Analyse und Ausbildung von Handlungen ,mehr oder weniger komplexe Handlungen in Teilhandlungen (Handlungsschritte) zu zerlegen, die genauer analysiert bzw. speziell ausgebildet werden müssen, damit die ganzheitliche Handlung angeeignet werden kann“" (Kossakowski und Lompscher 1988, S. 39).

An dieser Stelle sei darauf hingewiesen, dass Schülerhandlungen aus einer tätigkeitstheoretischen Perspektive vor allem hinsichtlich der Aneignung von Wissen und Können betrachtet werden. In diesem Fall ist auch von Aneignungshandlungen oder Lernhandlungen die Rede. Dabei wird der Lernprozess fokussiert. Lernhandlungen im Rahmen eines Lernprozesses lassen sich jedoch in Testaufgaben, wie sie hier zum Einsatz kommen, kaum abbilden. Stattdessen werden hier Lernprodukte betrachtet, d. h. der Fokus wird darauf gelegt, welche Handlungen sich die Lernenden bereits angeeignet haben und ggf. in einer gestellten Aufgabe zeigen und welche noch nicht. Wir gehen in unserem Ansatz davon aus, dass die im nachfolgend erläuterten Begriffssystem von Bruder und Brückner differenzierten Schülerhandlungen nicht nur Aneignungshandlungen darstellen, sondern auch dafür geeignet sind, im Rahmen von schriftlichen Tests das angeeignete Wissen und Können zu diagnostizieren. Dies erfolgt durch eine Analyse, welche Handlungen die Lernenden in Verbindung mit einem bestimmten mathematischen Inhalt bereits erfolgreich ausführen können.

Die wesentlichen Bausteine des Begriffssystems mit den entsprechenden Definitionen sollen hier referiert werden: „Der Aufbau des Begriffssystems erfordert zunächst geeignete Grundbegriffe (Operationen), die auch als Bausteine komplexer Handlungen praktikabel sind, die pädagogisch-psychologische Einsichten wider-

\footnotetext{
${ }^{1}$ einen entsprechenden Katalog siehe https:/www.standardsicherung.schulministerium.nrw.de/abiturgost/fach.php?fach=2, Aufruf am 24.03.2015.
} 
Tab. 1 Elementaroperationen nach Bruder und Brückner (1989)

Erfassen der Beziehungen von Teil und Ganzen

Zergliedern in Teile oder Strukturkomponenten eines Ganzen (i. S. von Zerlegen, Aufteilen)

Ausgliedern von Teilen oder Strukturkomponenten eines Ganzen (i. S. von Auswählen und Hervorheben bestimmter Teile eines zergliederten Ganzen gegenüber den anderen Teilen)

Inbeziehungsetzen von Teilen mit Feststellen der Identität oder Unterschiedlichkeit der untersuchten Teile

Zusammenfügen von Teilen zu einem neuen Ganzen (i. S. eines Zusammensetzens, Herstellen einer neuen Struktur bzw. Ganzheit)

Ersetzen von Teilen in verschiedenen Ganzen (i. S. eines Austausches bedeutungsgleicher, äquivalenter Teile oder formaler Einsetzungen)

Erfassen der Beziehungen von Objekt und Eigenschaft

Ausgliedern von Eigenschaften (i. S. von Auswählen und Hervorheben bestimmter wahrgenommener, vorgestellter oder aus der abstrakten Objektkenntnis gefolgerten Eigenschaften/Merkmale)

Inbeziehungsetzen von Eigenschaften bzw. von Objekt und Eigenschaft (ausgegliederte Eigenschaften werden auf Übereinstimmung geprüft bzw. ein Objekt wird bez. des Zutreffens oder Nichtzutreffens einer Eigenschaft untersucht)

spiegeln, die möglichst elementar und voneinander unabhängig sind [...]. Die von Lompscher gekennzeichneten grundlegenden analytisch-synthetischen Operationen des Erfassens der Beziehungen von Teil und Ganzem und Ding und Eigenschaft (im folgenden kurz Elementaroperationen genannt) erweisen sich als Elementarbausteine geeignet, ein Begriffssystem für Schülertätigkeiten beim Aufgabenbearbeiten zu bilden.“"(Bruder und Brückner 1989, S. 75).

Die theoretische Grundlage dieses Begriffssystems bilden also Elementaroperationen zum Erfassen der Beziehungen zwischen Teil und Ganzem sowie der Beziehungen von Objekt und Eigenschaft (s. Tab. 1). Wir führen die von Bruder und Brückner (1989) beschriebenen Elementaroperationen hier auf, um die gestufte Begriffsbildung von den Elementaroperationen über "Komplexe Denkoperationen“ bis zu den „Elementaren Aneignungshandlungen“ (Elementarhandlungen) beispielhaft nachvollziehbar zu machen.

Mit diesen Elementaroperationen lassen sich „Komplexe Denkoperationen“ beschreiben wie Vergleichen, Ordnen, Abstrahieren usw. Vergleichen wird gedeutet als „Inbeziehungsetzen von (ausgegliederten) Teilen oder Eigenschaften von Objekten mit dem Ziel, Übereinstimmung bzw. Unterschiedlichkeit bzgl. einer bestimmten (gegebenen) Eigenschaft festzustellen" (Bruder und Brückner 1989, S. 78). Ausgewählte „Komplexe Denkoperationen“ lassen sich wiederum zu den beiden Elementarhandlungen Identifizieren und Realisieren zusammen führen. Unter Identifizieren wird das ,Vergleichen der aufgenommenen Informationen zu Teilen oder Eigenschaften eines Objektes mit den Merkmalen bestimmter aktualisierter Abbilder (Stoffelemente, Handlungsvorschriften...) und Feststellung von Übereinstimmung oder Nichtübereinstimmung auf der Grundlage eines den jeweiligen Abbildungsmerkmalen entsprechenden Idealisierens der gegebenen Objektsituation" (S. 79) verstanden, während das Realisieren das „Transferieren, Konkretisieren oder Spezialisieren eines vorgegebenen (bzw. identifizierten) Handlungsgegenstandes (Stoffelemente, Vorgehensstrategien,...) auf eine gegebene Objektsituation und Zusammenfügen der so erzeugten Teile zu einem neuen Ganzen“" (S. 79-80) beinhaltet. 
Beispielsweise kann die erarbeitete Definition für eine Funktion (z. B. als eindeutige Zuordnung) mit der Elementarhandlung Identifizieren erschlossen werden, indem von gegebenen Objekten entschieden werden soll, ob es sich um eine Funktion handelt oder nicht. Gleichzeitig eignen sich Handlungsaufforderungen zum Identifizieren zum (teilweisen) Erfassen der Kompetenzfacette „Kenntnis des Funktionsbegriffs". Indem diagnostiziert wird, inwieweit Schülerinnen und Schüler innerhalb gegebener Zuordnungen Funktionen identifizieren können, können Rückschlüsse auf ein elementares Verständnis des Funktionsbegriffs bzw. der Funktionsdefinition gezogen werden.

Eine Realisierungsaufforderung wäre z. B. die Angabe eines eigenen Beispiels für eine Funktion - auch in verschiedenen Repräsentationen - oder die Veränderung einer gegebenen Darstellung so, dass sie eine Funktion beschreibt. Hier muss über Vergleichsoperationen hinausgegangen werden. Während das Identifizieren mit einer Entscheidung abschließt, die auch nicht explizit nach außen begründet werden muss, erfordert das Realisieren zusätzlich Konkretisierungen als spezifische komplexe Denkoperationen. Nach diesem Begriffsverständnis schließt erfolgreiches Realisieren Identifizierungshandlungen mit ein, geht aber noch deutlich darüber hinaus. Insofern wird eine auf Art und Umfang erforderlicher Elementaroperationen bezogene grundsätzliche strukturelle Hierarchie zwischen Identifizieren und Realisieren angenommen.

Dieser Unterschied zwischen Identifizieren und Realisieren wird in dem gewählten Modell damit als strukturell unabhängig von der Art des Gegenstandes angenommen, auf den die Handlung gerichtet ist. Im Fall der von uns untersuchten Items sind es die verschiedenen Darstellungswechsel funktionaler Zusammenhänge, d. h. es spielt von der Art der Handlungsstruktur her keine Rolle, ob es sich dabei z. B. um einen Darstellungswechsel zwischen Graph und Gleichung oder zwischen Tabelle und Graph handelt - s. Kap. 3. Diese strukturelle Inhaltsunabhängigkeit der Elementarhandlungen wird anhand von Itemschwierigkeiten jedoch nur dann ansatzweise erkennbar werden, wenn die gewählten Handlungsgegenstände eine vergleichbare innere Komplexität und Bekanntheit aufweisen. Andernfalls könnten die inhaltsbezogenen Schwierigkeiten, die also im Gegenstand selbst liegen, den Handlungsaspekt überlagern.

Die Trennung der beiden Elementarhandlungen Identifizieren und Realisieren lässt sich in ähnlicher Weise auch in der englischsprachigen Literatur finden. Die Unterscheidung von Leinhardt et al. (1990) zwischen interpretation und construction im Zusammenhang mit Aufgaben aus dem Bereich funktionaler Zusammenhänge hat deutliche Parallelen zu unserem Ansatz der Unterscheidung zwischen Identifizieren und Realisieren. Unter interpretation verstehen sie ,the action by which a student makes sense or gains meaning from a graph (or a portion of a graph), a functional equation, or a situation“ (Leinhardt et al. 1990, S. 8). Die Handlung construction umschreiben sie wie folgt: „By construction we refer to the act of generating something new. Construction refers to building a graph or plotting points from data (or from a function rule or a table) or to building an algebraic function for a graph." (Leinhardt et al. 1990, S. 12). Demnach stimmt construction mit dem hier beschriebenen Realisieren vollständig überein. Interpretation umfasst hingegen mehr als eine Identifizierungshandlung, da eine Interpretation meistens aus mehreren kognitiven Teil- 
handlungen besteht. Soll z. B. das Ergebnis einer Modellierung interpretiert werden, muss das mathematische Resultat mit dem realen Resultat in Verbindung gebracht werden (Identifizieren, Verknüpfen) und diese Verbindung muss im Allgemeinen auch kommuniziert werden (Beschreiben). Das Identifizieren hingegen bezieht sich auf eine deutlich elementarere Ebene, sodass eine detailliertere Aufschlüsselung der zur Aufgabenbearbeitung nötigen oder verwendeten kognitiven Teilhandlungen möglich ist.

Des Weiteren findet sich bei Dreher et al. (2014) eine Kompetenzstrukturmodellierung zu Darstellungswechseln in der Bruchrechnung, die auch eine ähnliche Unterscheidung der Handlungen vornehmen. Sie differenzieren zwischen den Handlungen examine, perform und justify. Die ersten beiden Handlungen kommen den hier betrachteten Handlungen Identifizieren und Realisieren sehr nahe. Dreher et al. konnten ein dreidimensionales Kompetenzstrukturmodell mit Mehrfachladungen empirisch bestätigen. Demnach fordern die Aufgaben zu Darstellungswechseln entweder ein examining oder ein performing. Zusätzlich kann noch eine Begründung (justifying) gefordert sein.

Unser Ansatz unterscheidet sich insofern von Dreher et al., als dass wir aufgrund der postulierten strukturellen Zuammenhänge der beiden Handlungen Identifizieren und Realisieren davon ausgehen, dass die Handlung Identifizieren grundlegend ist für die Bewältigung nahezu aller Anforderungen. Ohne die Identifikation des Handlungsgegenstandes kann eine passende Realisierungshandlung nicht initiiert werden. Realisierungshandlungen besitzen eine größere Komplexität (im Sinne einer Mehrschrittigkeit) als Identifizierungshandlungen. Das Realisieren enthält zusätzliche Anforderungen (s. Definition), nämlich ein Konkretisieren bzw. Spezialisieren des identifizierten Handlungsgegenstandes im Sinne von etwas „erzeugen“ oder „herstellen“. Das Ergebnis dieses „Herstellungsanteils“ können z. B. verbale Formulierungen, graphische Darstellungen und gelöste Gleichungen sein. Fehlerhafte Aufgabenbearbeitungen mit Realisierungsanforderung zeigen entweder Fehler im Identifikationsanteil (Zuordnung von Begriffsinhalten zur Situation, Entscheidung der Passung eines bekannten Verfahrens und deren Auswahl) oder im „Herstellungsanteil"“ (beim Aufschreiben eines Terms, einer konkreten Berechnung, Zeichnung eines Graphen) oder sogar in beidem.

Die fachdidaktische Bedeutung einer Unterscheidung zwischen Identifizieren und Realisieren als relevanten Schüleraktivitäten in der hier erfolgten hierarchischen Form ergibt sich insbesondere aus den Erwartungen an einen verständigen Umgang mit mathematischen Verfahren im Gegensatz $\mathrm{zu}$ einem reinen Abarbeiten eines Schemas. Ein verständiger Umgang mit Kalkülen (und Begriffen) erfordert u. a. auch ein Prüfen von deren Anwendbarkeit auf eine gegebene Situation. Ein Verzicht auf das Identifizieren als immanente Prüfoperation bei der theoretischen Modellierung des Realisierens und bei der entsprechenden Itemkonstruktion würde nicht den aktuellen Zielvorstellungen eines allgemeinbildenden Mathematikunterrichts entsprechen.

Aufbauend auf den Elementarhandlungen und diese erweiternd werden im Modell von Bruder und Brückner fünf Grundhandlungen beschrieben: Erkennen, 
Beschreiben, Verknüpfen, Ausführen ${ }^{2}$ und Begründen. Grundhandlungen und Elementarhandlungen stehen in einem hierarchischen Abhängigkeitsverhältnis. Grundhandlungen liegen aus theoretischer Sicht in ihrem Anforderungsprofil in der Hierarchie (nach Komplexität der Handlungsanforderungen) über den Elementarhandlungen. Die Grundhandlungen beschreiben auch Vernetzungen von Lerninhalten und bereiten deren schrittweise Übertragbarkeit durch die unterschiedlichen Perspektiven auf den Lerninhalt vor. Aufgrund dieser in der theoretischen Perspektive angelegten Hierarchie von Handlungen besteht die Vermutung, dass unser Ansatz auch für Kompetenzstufenmodelle eine Rolle spielen kann (siehe auch Kap. 3.3). Diese Vermutung äußern auch Dreher et al. (2014). Sie betrachteten die empirischen Itemschwierigkeiten und konnten eine Abstufung der Handlungen examine und perform für den Inhaltsbereich Bruchrechnung nachweisen. Dabei unterschieden sie Aufgaben, die zusätzliche Begründungsaufforderungen enthalten von denjenigen Aufgaben, die eine solche Anforderung nicht enthalten. In beiden Fällen weisen diejenigen Aufgaben, die die Handlung examining fordern, eine niedrigere Itemschwierigkeit auf als diejenigen Aufgaben, die die Handlung performing fordern.

In der SOLO (Structure of the Observed Learning Outcome) taxonomy, die auf Biggs (Biggs und Tang 2007) zurückgeht und learning outcomes aufgrund verschiedener Komplexitätsstufen klassifiziert, werden die einzelnen Stufen mithilfe von Handlungen beschrieben:

- Unistructural: Identify, Name, Follow simple procedures

- Multistructural: Combine, Describe, Enumerate, Perform serial skills, List

- Relational: Analyze, Apply, Argue, Compare/contrast, Criticize, Explain causes, Relate, Justify

- Extended Abstract: Create, Formulate, Generate, Hypothesize, Reflect, Theorize

Einige dieser Handlungen weisen Ähnlichkeiten $\mathrm{zu}$ den hier beschriebenen Elementar- und Grundhandlungen auf. So finden sich beispielsweise auf der ersten Ebene (unistructural) die beiden Handlungen Identify und Follow simple procedures, die sich den beiden Elementarhandlungen Identifizieren und Realisieren zuweisen lassen. Auf der nächsten Ebene finden sich die Handlungen Combine und Describe, die den beiden Handlungen Verknüpfen und Beschreiben nahe kommen. Auf der dritten Ebene lässt sich die Handlung Apply der Handlung Ausführen zuordnen, während die beiden Handlungen Argue und Justify der Grundhandlung Begründen nahe kommen. Es muss an dieser Stelle allerdings betont werden, dass Biggs die Handlungen nicht näher ausführt, die Zuordnungen können demnach nur aufgrund des Namens der Handlung und der zugehörigen Ebene erfolgen. Dennoch liefert das Modell von Biggs Hinweise darauf, dass durch die Differenzierung verschiedener Handlungen Stufenmodellierungen erfolgen können. Insgesamt bilden die Elementarhandlungen und die Grundhandlungen in Verbindung mit den drei mathematischen Inhaltskategorien Begriffe, Zusammenhänge und Verfahren ein Begriffssystem, mit dem sich eine Vielzahl an Lösungswegen bzw. Erwartungs-

\footnotetext{
${ }^{2}$ Gegenüber dem Originalmodell bei Bruder und Brückner wurde eine Wortmarke ausgetauscht (Ausführen statt Anwenden), um Missverständnisse zu vermeiden, die sich aus dem etablierten Alltags- oder Fachsprachgebrauch der Wortmarke „Anwenden“ ergeben könnten.
} 
Tab. 2 Definition der Grundhandlungen Beschreiben und Begründen nach Bruder und Brückner (1989)

Beschreiben als syntaktische Darstellung von erzeugten oder im Gedächtnis gespeicherten Abbildern erfordert Identifizieren und Realisieren einer dem gespeicherten (oder erzeugten) Abbild adäquaten umgangssprachlichen Formulierung oder Darstellung in mathematischer Terminologie und Symbolik und Entäußerung der Informationen auf sprachlicher oder materialisierter Ebene.
Begründen als Nachweis der Wahrheit oder Falschheit einer gegebenen Aussage mit gegebenen/bekannten Normativen oder Sätzen und Verfahren bzw. Rechtfertigung einer Behauptung, Annahme u. ä. erfordert:

a.) Vergleichen eines vorgegebenen Sachverhaltes mit gegebenen bzw. bekannten Normativen

b.) Realisieren gegebener bzw. realisierter bzw. identifizierter elementarer Beweisverfahren

horizonten zu Mathematikaufgaben differenziert und zumindest teilstandardisiert beschreiben lassen.

Beispielsweise kann die erfolgreiche Aneignung eines mathematischen Verfahrens diagnostiziert werden, wenn zu diesem Verfahren Identifizierungs- und Realisierungshandlungen mit entsprechenden Aufgaben gefordert werden. Dabei umfasst eine Identifizierungshandlung zu einem mathematischen Verfahren Prüfoperationen, ob das vorgegebene Verfahren auf die gegebene Situation überhaupt anwendbar ist. Muss erst ein Verfahren ausgewählt im Sinne von ,gefunden“werden, das auf die gegebene Situation passt, sind mehrschrittige bzw. mit Transfer behaftete Schülerhandlungen erforderlich. In diesem Fall würden wir von Erkennen der Anwendbarkeit eines Verfahrens sprechen als nächster Stufe, da neben dem „einfachen“ Identifizieren als einem „In-Beziehung-Setzen“ von Informationen auch ein Auswahlprozess erforderlich ist. Realisierungshandlungen z. B. zu einem mathematischen Verfahren umfassen zunächst das Abarbeiten der Teilschritte des gegebenen Verfahrens, können aber auch bis zum „Passendmachen“ des Verfahrens auf eine gegebene Situation reichen. In diesem Fall würden wir nicht mehr nur elementar von Realisieren sprechen, sondern in einem komplexeren Sinne von Ausführen. Komplexe Lösungswege erfordern ggf. spezifische Verknüpfungen von Grundhandlungen und Inhalten, die wiederum auch mit anderen Wortmarken beschrieben werden können, z. B. als Such- und Planungshandlungen für Lösungswege usw. (ebenda).

Wir konzentrieren uns im Folgenden auf die Darstellung der beiden Grundhandlungen Beschreiben und Begründen, da diese Grundhandlungen im anvisierten Inhaltsbereich besonders relevant erscheinen und bereits in der Itemkonstruktion der in Kap. 3 dargestellten Untersuchung fokussiert wurden (s. Tab. 2). Auch an dieser Stelle unterscheiden wir uns von Dreher et al., da dort - zusätzlich zu examining und performing - lediglich die Handlung Justifying aufgenommen wurde, die einer Begründungsaufforderung entspricht. Beschreibungshandlungen werden demnach dort nicht explizit unterschieden.

Die vorgestellten Definitionen der jeweiligen Handlungsstruktur beziehen sich auf eine geringe Komplexität der gestellten Anforderungen. Beim Begründen z. B. sind damit die Grundtypen für Begründungen gemeint (vgl. Bruder und Pinkernell 2011): Identifizieren und Realisieren eine Begriffes, eine Satzes oder eines Verfahrens; Argumentation mit Gegenbeispiel oder Argumentation nach Kontraposition mit jeweils einem Begriff, Satz oder Verfahren.

Am Beispiel des Funktionsbegriffs könnte eine Beschreibungsaufforderung darin bestehen, dass ein Schüler seine Vorstellungen von einer Funktion in eigenen Worten 
formulieren soll, ggf. auch für einen bestimmten Adressaten. Hier sind qualitativ sehr unterschiedliche Beschreibungen denkbar. Diese sind unter anderem abhängig von den aufgebauten Grundvorstellungen ${ }^{3}$ (unter Bezug auf Vollrath (1989) sind das die Zuordnungsvorstellung, die Kovariationsvorstellung und die Objektvorstellung) zum Funktionsbegriff. Beispielsweise resultieren auf Basis adäquater Grundvorstellungen zum Funktionsbegriff andere Beschreibungen als auf Basis einer ggf. auswendig gelernten Definition. Darüber hinaus ist das Beschreibungsergebnis abhängig von den Verbalisierungsfähigkeiten der Lernenden. Eine Begründungsaufforderung erfordert hingegen immer einen Argumentationsprozess, wenn beispielsweise der Schüler dazu aufgefordert wird, eine Begründung zu liefern, warum es sich bei einem vorliegenden Beispiel um eine Funktion handelt oder nicht. Hierfür müssen definierende Elemente des Funktionsbegriffs herangezogen werden. Eine gewisse Verbalisierungsanforderung besteht auch beim Begründen, nur ist sie stärker an der Fachsprache orientiert, d. h. die Zulässigkeit der Argumente ist stärker normiert als beim Beschreiben.

Die konkrete Ausrichtung der vier Handlungen Identifizieren, Realisieren, Beschreiben und Begründen innerhalb des Bereichs Darstellungswechsel wird im nachfolgenden Kapitel dargestellt.

\section{Schülerhandlungen im Inhaltsbereich Darstellungswechsel funktionaler Zusammenhänge}

\subsection{Theoretisches Modell zu den Handlungen in HEUREKO}

Im Folgenden wird eine von uns vorgenommene Reanalyse des Datensatzes aus dem Projekt HEUREKO unter tätigkeitstheoretischer Perspektive vorgestellt. Hierfür wurden bereits vor der Itemkonstruktion von HEUREKO vier Handlungen aus den Elementarhandlungen und Grundhandlungen ausgewählt, die im Themenfeld der funktionalen Zusammenhänge aus theoretischer Perspektive im fokussierten Inhaltsbereich geeignet erschienen: Identifizieren, Realisieren, Beschreiben und Begründen. Es handelt sich hierbei um diejenigen Handlungen, die bei einem Darstellungswechsel im Vordergrund stehen und die zur Bewältigung einer Vielzahl von Anforderungen in diesem Inhaltsbereich erforderlich sind.

Das Identifizieren beim Umgang mit Darstellungsformen und -wechseln umfasst ein vergleichendes Inbeziehungsetzen bedeutsamer Werte und Eigenschaften einer Funktion in einer bestimmten Darstellungsform und das Zuordnen zweier Darstellungsformen, die die gleiche mathematische Funktion darstellen.

Das Realisieren erfordert die Konstruktion neuer Elemente, die nicht gegeben sind. Hier bedeutet dies die Durchführung eines Darstellungswechsels, indem zu einer gegebenen Startrepräsentation die gewünschte Zielrepräsentation erstellt wird.

Neben diesen beiden Elementarhandlungen berücksichtigen wir die beiden Grundhandlungen Beschreiben und Begründen: Beim Beschreiben werden die Lernenden dazu aufgefordert, ihren Lösungsweg verbal darzulegen. Das Beschreiben

${ }^{3}$ Zum Konzept der Grundvorstellungen vgl. vom Hofe (1995). 
Abb. 2 Beispielaufgabe zum Identifizieren, Realisieren und Beschreiben
Gegeben ist folgender Graph:

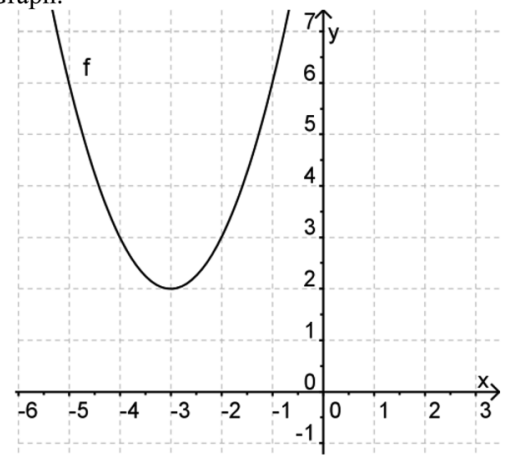

1. Stelle die Gleichung für die verschobene Normalparabel $\mathrm{f}$ auf.

2. Beschreibe wie du vorgegangen bist.

erfolgt entweder direkt nach dem Realisieren eines Darstellungswechsels oder die Lernenden werden aufgefordert, ihren Lösungsweg zu skizzieren, ohne dabei tatsächliche Berechnungen durchzuführen. Beim Begründen müssen die Lernenden entweder nach einer Identifizierungshandlung die Korrektheit der eigenen Lösung argumentativ darlegen oder erläutern, warum eine gegebene Lösung zu einem Darstellungswechsel richtig oder falsch ist. Um die strukturellen Zusammenhänge und Unterschiede der von uns ausgewählten Handlungen im Bereich Darstellungswechsel funktionaler Zusammenhänge zu verdeutlichen, werden im Folgenden zwei Beispielaufgaben vorgestellt.

Die Aufgabe in Abb. 2 enthält zwei Teilaufgaben, denen unterschiedliche Kombinationen der vier Handlungen zugeordnet werden können. Im ersten Teil ist das Aufstellen der Gleichung der mathematischen Funktion gefordert, die in graphischer Darstellungsform vorliegt. Da die Zieldarstellung (Gleichung) konstruiert werden muss, ist hier ein Realisieren gefordert. Um die Realisierungshandlung auszuführen, müssen aber zunächst die wesentlichen Eigenschaften der mathematischen Funktion aus dem gegebenen Graphen abgelesen werden. Dies erfordert eine Identifizierungshandlung. Demnach enthält diese Teilaufgabe die Handlungen Identifizieren und Realisieren. In der zweiten Teilaufgabe ist die Beschreibung des Lösungsweges gefragt. Für eine solche Beschreibung ist ein Überblick über die Realisierung des Darstellungswechsels notwendig. Gleichzeitig benötigt die Realisierung ein vorheriges Identifizieren (wie bei Teilaufgabe 1). Folglich sind zur Lösung der zweiten Teilaufgabe die Handlungen Identifizieren, Realisieren und Beschreiben notwendig.

Die Aufgabe in Abb. 3 erfordert eine Begründungshandlung. Zur Aufgabenlösung müssen zunächst wesentliche Eigenschaften der repräsentierten mathematischen Funktion identifiziert werden, um eine der beiden Gleichungen auszuwählen. Des Weiteren ist eine Begründung dieser Entscheidung gefordert. Demnach sind zur Lösung dieser Aufgabe die Handlungen Identifizieren und Begründen erforderlich.

Aufgrund der Struktur der Handlungen wird folgendes theoretisches Modell postuliert: Die beiden Elementarhandlungen stellen unterschiedliche Anforderungsstrukturen dar und lassen sich demnach nicht zu einer gemeinsamen Handlung zusammenfassen. Bei einem Darstellungswechsel müssen zunächst die wesentlichen Eigenschaften einer Funktion innerhalb einer gegebenen Darstellungsform identi- 
Gegeben sind die folgende Wertetabelle und zwei Gleichungen:

\begin{tabular}{|l|c|c|c|c|}
\hline$x$ & 0 & 1 & 2 & 3 \\
\hline$y$ & 2 & 3 & 6 & 11 \\
\hline
\end{tabular}

$$
y=2 x+2
$$

(2) $y=x^{2}+2$

Entscheide welche Gleichung zu der Wertetabelle passt.

Antwort:

Begründe deine Entscheidung:

Abb. 3 Beispielaufgabe zum Identifizieren und Begründen

fiziert werden. Demnach ist zur Lösung aller Aufgaben in diesem Bereich eine Identifizierungshandlung notwendig. Um daran anknüpfend einen Darstellungswechsel zu realisieren, müssen zusätzlich die wesentlichen Eigenschaften einer Funktion in der Zieldarstellung geeignet repräsentiert werden. Hierzu muss die anvisierte Darstellungsform zunächst konstruiert werden, beispielsweise muss bei der graphischen Darstellungsform ein Koordinatensystem gezeichnet werden, die Achsen müssen geeignet beschriftet und eingeteilt werden. In einem nächsten Schritt wird dann der Funktionsgraph gezeichnet, indem beispielsweise bei einer linearen Funktion der $\mathrm{y}$-Achsenabschnitt als ein Punkt auf dem Graphen markiert wird und dann mithilfe eines Steigungsdreiecks ein zweiter Punkt markiert wird, um mithilfe dieser beiden Punkte den Graphen zeichnen zu können. Eine Realisierungshandlung erfordert demnach neben dem Identifizieren zusätzliche Anforderungen.

Die beiden Grundhandlungen Beschreiben und Begründen werden in der vorliegenden Studie zu einer gemeinsamen Handlung Beschreiben und Begründen zusammengefasst, da diese beiden Grundhandlungen in Zusammenhang mit Darstellungswechseln nur geringe Unterschiede hinsichtlich der Anforderungsstruktur aufweisen und die Verbalisierung eine dominante Rolle spielt. Das lässt sich damit erklären, dass sich das Begründen im anvisierten Inhaltsbereich meistens auf das Ergebnis der Aufgabenbearbeitung bezieht. In anderen Themenbereichen ist es auch denkbar das Begründen auf den Lösungsweg zu beziehen, beispielsweise bei einer Beweisaufgabe. Dies ist jedoch im Bereich von Darstellungswechseln selten der Fall, sodass diese Form des Begründens hier außer Acht gelassen wurde. Das Begründen im Sinne eines Auswählens zulässiger Argumente und Schlussweisen wird im vorliegenden Itemsatz nur auf einem niedrigen Niveau gefordert und die Verbalisierung steht im Vordergrund. Gleichzeitig ist diese Verbalisierung weder beim Identifizieren noch beim Realisieren gefordert, sodass das Beschreiben und Begründen nicht mit diesen Handlungen gleich gesetzt werden kann.

\subsection{Empirische Überprüfung des resultierenden Messmodells}

\subsubsection{Das resultierende Messmodell}

In einem nächsten Schritt wurde aus den theoretischen Überlegungen zur Anforderungsstruktur der im gewählten Gegenstandsbereich relevanten Handlungen 


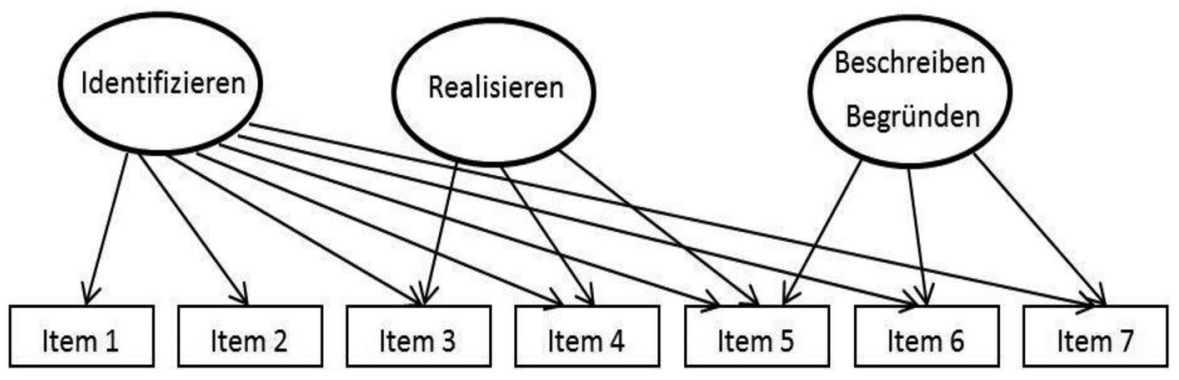

Abb. 4 Modellstruktur mit Mehrfachladungen

ein resultierendes Messmodell abgeleitet, das die strukturellen Zusammenhänge und Unterschiede der Handlungen abbildet.

Die relevanten Handlungen werden als drei Handlungsdimensionen aufgefasst: Identifizieren, Realisieren sowie Beschreiben und Begründen. Da sich ein Item im Allgemeinen auf mehrere Handlungen bezieht (vgl. die Beispielaufgaben in Abb. 2 und 3) wurden Mehrfachladungen zugelassen (s. Abb. 4).

Um eine solche komplexe Ladungsstruktur abzubilden, sind theoretisch fundierte Vorannahmen über die Eigenschaften der Aufgaben und die inhaltliche Bedeutung der latenten Variablen wesentlich (Hartig und Höhler 2010). Nach der Definition der drei Handlungsdimensionen im theoretischen Modell laden alle Items auf die Dimension Identifizieren. Das Realisieren ist in solchen Items gefordert, in denen die Zieldarstellung konstruiert werden muss und/oder der Lösungsweg beschrieben werden muss. ${ }^{4}$ Hingegen ist es nicht explizit in jenen Items gefordert, in denen eine Begründung der (gegebenen) Lösung gefordert ist, da hier nicht der Lösungsweg, sondern das Ergebnis fokussiert wird. Alle Items, in denen entweder eine Beschreibung des Lösungsweges oder eine Begründung der (gegebenen) Lösung gefordert ist, laden auf die Dimension Beschreiben und Begründen.

Die Handlung Identifizieren entspricht demnach einer Art Generalfaktor (vgl. Hartig und Höhler 2010), der notwendig ist, um die gemeinsamen Anforderungen aller Aufgaben zu Darstellungswechseln zu bewältigen. Das Realisieren bzw. das Beschreiben und Begründen hingegen beschreiben spezifische Anforderungen, die zusätzlich zur Aufgabenbearbeitung erforderlich sind. Hartig und Höhler weisen darauf hin, dass die Interpretation solcher Modelle mit Mehrfachladungen schwieriger ist als bei Modellen mit Einfachstruktur und immer unter Berücksichtigung der komplexen Ladungsstruktur erfolgen muss. Neben dem von uns postulierten Modell haben wir folgende alternativen Modelle in Betracht gezogen, die aus theoretischer Perspektive plausibel erscheinen: Das 1-dimensionale Vergleichsmodell (1D) basiert auf der Annahme, dass sich keine Unterschiede in den Handlungen zeigen und alle Handlungen zu einer Dimension zählen. Demnach besitzen alle Handlungen die gleiche Anforderungsstruktur im Bereich Darstellungswechsel. Die Handlungen

\footnotetext{
${ }^{4}$ Dieser Zuordnung liegt die Annahme zugrunde, dass zur Beschreibung des Lösungsweges ein Überblick über die Realisierung des Darstellungswechsels notwendig ist. Demnach laden die Items, in denen eine Beschreibung erforderlich ist, auch auf die Dimension Realisieren
} 
unterscheiden sich in diesem Fall lediglich hinsichtlich ihrer Komplexitätsniveaus. Sie stellen zwar unterschiedliche Ausprägungen, aber dieselbe Kompetenzfacette dar.

In einem 2-dimensionalen Vergleichsmodell (2D - IR) werden die beiden Handlungen Identifizieren und Realisieren zu einer Dimension zusammengefasst, da diese die beiden elementaren Handlungen darstellen. Dieser Dimension werden alle Items zugeordnet, da unabhängig von der genauen Aufgabenstellung immer eine spezifische Kombination von Identifizierungs- und Realisierungshandlungen erfolgen muss, um ein Item korrekt zu lösen. Bei der Durchführung eines Darstellungswechsels ist immer auch die Identifizierung wesentlicher Eigenschaften gefordert, die im Laufe der Aufgabenbearbeitung mehrere Male erfolgen muss, um die Korrektheit des Ergebnisses zu gewährleisten. Beim Identifizieren zweier Darstellungsformen, die die gleiche mathematische Funktion darstellen, muss auch ein Überblick über die Realisierung eines Darstellungswechsels vorliegen, um die Darstellungsformen einander zuzuordnen. Die zweite Dimension enthält alle Items, die eine Beschreibung oder Begründung fordern, da dort die sprachliche Komponente im Vordergrund steht. Beim Identifizieren und Realisieren ist keine Verbalisierung erforderlich, sodass hier entsprechend dem theoretischen Modell eine andere Anforderungsstruktur vorliegt.

In einem weiteren 2-dimensionalen Vergleichsmodell (2D - I) stellt die Handlung Identifizieren die erste Dimension dar. Dieser Dimension werden alle Items zugeordnet, da davon ausgegangen wird, dass immer eine Identifizierungshandlung notwendig ist, um ein Item korrekt zu lösen. Die Handlungen Realisieren, Beschreiben und Begründen werden zur zweiten Dimension zusammengefasst, da bei diesen Handlungen immer eine zusätzliche Komponente hinzukommt, die vom Aufgabenbearbeiter erzeugt (hergestellt) werden muss -entweder die Zieldarstellungsform oder eine Verbalisierung.

Im 4-dimensionalen Modell (4D) wird angenommen, dass sich alle vier Handlungen voneinander trennen lassen und demnach vier unterschiedliche Dimensionen darstellen. Dabei werden ähnlich wie im 3-dimensionalen Modell Mehrfachladungen zugelassen. Der einzige Unterschied zum 3-dimensionalen Modell besteht darin, dass hier die beiden Handlungen Beschreiben und Begründen separate Dimensionen darstellen. Alle Items, in denen eine Begründung der (gegebenen) Lösung gefordert ist, laden auf die Dimension Begründen. Da für eine Begründung auch die wesentlichen Werte bzw. Eigenschaften identifiziert werden müssen, laden die Items - wie im 3-dimensionalem Modell - zusätzlich auf die Dimension Identifizieren. Diejenigen Items, in denen der Lösungsweg beschrieben werden soll, laden auf die Dimension Beschreiben. Für eine Beschreibung der Lösung sind zusätzlich Identifizierungs- und Realisierungshandlungen notwendig, sodass die entsprechenden Items - auch analog zum 3-dimensionalen Modell - zusätzlich auf die Dimensionen Identifizieren und Realisieren laden.

\subsubsection{Methode}

In HEUREKO wurden insgesamt 278 mathematische Aufgaben zum Themenfeld Funktionen konstruiert, die sich jeweils aus einem Darstellungswechsel in Kombination mit bestimmten Handlungen zusammensetzen. Im Haupttest wurden insgesamt 120 der konstruierten und anhand der Ergebnisse der Pilotierung über- 
arbeiteten Aufgaben eingesetzt. Diese wurden auf vier verschiedene Testhefte in Form eines Multi-Matrix-Designs aufgeteilt (Gonzalez und Rutkowski 2010). Zusätzlich wurde zu einem späteren Zeitpunkt ein weiterer sogenannter Kurztest eingesetzt, der Ankeritems enthielt, um die Testhefte miteinander zu verbinden. ${ }^{5}$ Jedes Testheft enthielt 30 Aufgaben, die Testdauer betrug $40 \mathrm{~min}$. An der Studie nahmen insgesamt 27 Klassen teil, davon 9. (19) und 10. (8) Klassen von acht Gymnasien aus dem Raum Südhessen. Die Stichprobe des Haupttests bestand aus insgesamt $N=650$ Schülerinnen und Schülern (451 9. Klässler und 199 10. Klässler), wobei fünf Schülerinnen und Schüler aufgrund unseriöser Bearbeitungen nicht in die Analyse aufgenommen wurden. Die Ergebnisse des Haupttests wurden anhand vorgegebener Kriterien evaluiert, hierbei wurden die Items dichotom $(0,1)$ kodiert (fehlende Werte wurden entsprechend mit einer 9 kodiert, siehe auch Fußnote 7).

Da es sich um eine Reanalyse des HEUREKO Datensatzes handelt, mussten für die nachfolgende empirische Untersuchung zu den Handlungen zunächst die 120 in HEUREKO eingesetzten Aufgaben neu kategorisiert werden (eine gemeinsame Modellierung der Darstellungswechsel und Handlungen war aufgrund des zu geringen Itemsatzes nicht möglich). Dabei wurde die Kategorisierung nicht nach Darstellungswechseln durchgeführt, sondern nach Handlungen, sodass jedem Item die entsprechenden Handlungen zugewiesen wurden. Da einige Aufgaben aus mehreren auswertungsrelevanten Items bestanden, ergab sich ein Itemsatz von 144 Items.

Die Modellierung erfolgte mittels probabilistischer Testmodelle (dem RaschModell) der Item Response Theorie (Moosbrugger und Kelava 2007). Dabei wurde ein ein-parameter-logistisches Modell nach Rasch (1960/1980) zugrunde gelegt. Zunächst wurde mit der Software CONQUEST 2.0 gearbeitet (Wu et al. 2007), da diese die Itemfit-Werte ausgibt. Unter der Annahme des theoretisch postulierten 3D Modells wurden Items mit schlechten Itemfitwerten eliminiert (Infit - weighted mean square $<0,8$ oder $>1,2$ und T-Wert $>1,96$ ).

Weitere Analysen erfolgten mit der Software Mplus (Muthén und Muthén 19982010). Dabei wurden die Ladungen der latenten Variablen auf 1 fixiert. Um die dimensionale Struktur zu überprüfen, wurde das postulierte 3-dimensionale Messmodell mit verschiedenen anderen Modellen (1-dimensional, 2-dimensional, ...) verglichen. Hierzu wurde das informationstheoretische Maß des Bayesian Information Criterion (BIC) verwendet. ${ }^{6}$ Das BIC enthält die Likelihood des angepassten Modells und bezieht die Anzahl der Modellparameter - in Abhängigkeit der Stichprobengröße - mit ein, um grundsätzlich einfache Modelle mit wenigen Parametern zu favorisieren. Je niedriger der BIC Wert, desto besser passt das Modell auf die Daten (Moosbrugger und Kelava 2007). Die Software MPlus liefert zusätzlich das adjustierte BIC, welches zwar auch Modelle mit weniger Parametern bevorzugt, dabei jedoch Modelle mit mehr Parametern weniger stark benachteiligt wie das BIC (Sclove 1987).

\footnotetext{
${ }^{5}$ Der Kurztest umfasste 12 Items. Diese stammten zu gleichen Anteilen aus den vier Testheften, d.h. die vier Testhefte wurden durch verschiedene gemeinsame Items miteinander verankert.

${ }^{6}$ Weitere Informationen über multidimensionale IRT Modellierungen finden sich beispielsweise bei Hartig und Höhler (2008) oder Walker und Beretvas (2003).
} 


\subsubsection{Ergebnisse}

Im Durchschnitt bearbeiteten die Probanden 19,31 (SD=5,22) der 30 Aufgaben eines Testhefts und davon wurden 7,62 ( $\mathrm{SD}=3,90)$ Aufgaben korrekt gelöst. ${ }^{7} \mathrm{Da}$ unser Test die Themen lineare und quadratische Funktionen beinhaltet und diese Themen zum Testzeitpunkt bereits in beiden Klassenstufen (9 und 10) unterrichtet wurden, haben wir alle Schülerinnen und Schüler einer Stichprobe zugeordnet. Im weiteren Verlauf des Unterrichts in Klasse 9 und 10 wurden diese beiden Funktionstypen nicht mehr explizit thematisiert, sodass wir davon ausgehen, dass die Leistungen in diesem Themenbereich durch den weiteren Unterricht nicht maßgeblich beeinflusst wurden. Gestützt wird diese Annahme durch die Feststellung, dass sich die Leistung der 9. Klassen nicht signifikant von der Leistung der 10. Klassen unterschied. Dies wurde mithilfe einer univariaten Varianzanalyse in SPSS überprüft $(\mathrm{F}(1,643)=0,126$, $p=0,722)$, wobei die Lösungsrate die abhängige Variable darstellte. Darüber hinaus haben wir die vorliegenden Daten auf Differential Item Functioning (DIF) (vgl. Baranowski et al. 2006) überprüft, da bei einem vorliegenden DIF die Zusammenlegung der beiden Teilstichproben der 9. und 10. Klassen als kritisch zu betrachten ist. Hierfür wurden die Ankeritems herangezogen. Insgesamt wurde eine DIF Analyse bei 12 Items mithilfe der Software CONQUEST 2.0 vorgenommen. Die Analyse zeigt im ersten Schritt, dass den 10. Klässlern die 12 Items im Mittel leichter fallen als den 9. Klässlern. Sie zeigen demnach höhere Leistungen (mit einer Differenz von 0,204 logits), wobei daraus noch kein vorliegendes DIF geschlussfolgert werden kann. In einem zweiten Schritt wurden die Wechselwirkungen zwischen den Itemschwierigkeiten und der Klassenstufe untersucht. Der Chi-Quadrat Test wird in diesem Fall nicht signifikant $(\mathrm{df}=12$; Sig Level $=1,000)$, sodass kein DIF vorzuliegen scheint. Beim Vergleich des Abschneidens der 9. und 10. Klässler bei den einzelnen Items fällt lediglich ein Item auf, dass eine relativ hohe Differenz aufweist $(0,61$ logits) und damit problematisch erscheint. Da es sich aber nur um ein einzelnes Item handelt, ist der Effekt als gering einzustufen (Adams und Wu 2010).

Als nächstes wurden mithilfe der Software Conquest unter der Annahme des theoretisch postulierten 3-dimensionalen Modells die Itemfitwerte betrachtet. Aufgrund eines schlechten Itemfitwertes mussten insgesamt 13 Items entfernt werden. Von den verbleibenden 131 Items zeigen 109 Items Werte zwischen 0,90 und 1,10, was einem sehr guten Itemfit entspricht.

Die Verteilung der Items auf die verschiedenen Handlungsdimensionen lässt sich Tab. 3 entnehmen. Die ungleiche Verteilung der Items auf die Dimensionen ergibt sich dadurch, dass das Identifizieren als grundlegend für alle Aufgabenbearbeitungen angenommen wurde (im Sinne eines Generalfaktors). Des Weiteren ist das Realisieren

\footnotetext{
${ }^{7}$ Die fehlenden Antworten wurden als missing kodiert. Um sicherzustellen, dass jeder Darstellungswechsel und jede Handlung von den Schüler/innen bearbeitet wurde, waren die Aufgaben im Testheft entsprechend gleichmäßig verteilt. Die Schüler/innen wurden aufgefordert, die Testhefte von vorne nach hinten durchzuarbeiten. Sie durften Aufgaben überspringen, wenn sie ihnen schwierig erschienen. Prinzipiell hätten die Schüler/innen auch alle Aufgaben ansehen und sich dann die leichten Aufgaben raussuchen können, das konnten wir aber nur ganz selten beobachten. Die meisten Schüler/innen bearbeiteten die Testhefte in der vorgegebenen Reihenfolge. Wir gehen hier also nur von geringen und damit vernachlässigbaren Effekten in Bezug auf die Itemschwierigkeit und die Itemfitwerte aus.
} 
Tab. 3 Anzahl der Items pro Dimension. (Die Auswahl eines within oder between Ansatzes ergibt sich hier jeweils aus theoretischen Betrachtungen, siehe 3.2.1.)

\begin{tabular}{lll}
\hline Dimension & Anzahl Items für 3D Modell (within) & Anzahl Items für 4D Modell (between) \\
\hline Identifizieren & 131 & 49 \\
Realisieren & 62 & 40 \\
Beschreiben & 42 & 22 \\
Begründen & & 20 \\
\hline
\end{tabular}

auch in Items notwendig, in denen eine Beschreibung gefordert ist. Demnach sind auf die Dimensionen Identifizieren und Realisieren mehr Items verteilt.

Die Item- und Personenparameterverteilung ist in Abb. 6 dargestellt und kann als zufriedenstellend angesehen werden. In den ersten drei Spalten ist die Verteilung der Personenparameter in Abhängigkeit von den Dimensionen abgebildet (Dimension 1=Identifizieren, Dimension 2=Realisieren, Dimension 3=Beschreiben und Begründen). In der vierten Spalte ist die Verteilung der Itemschwierigkeitsparameter dargestellt.

Die latenten Korrelationen sind in Tab. 4 unterhalb der Diagonalen dargestellt und können als hinreichend inkrementell angesehen werden. Eine negative Korrelation zwischen Identifizieren und Beschreiben/Begründen bedeutet, dass diejenigen Lernenden, die mehr Leistung bei Identifizieren-Items zeigen, gleichzeitig weniger Leistung bei Beschreiben- und Begründen-Items zeigen. Es handelt sich dabei um einen schwachen Zusammenhang.

Auf den Diagonalen sind die Verhältnisse der Varianzen (EAP/PV - expected a posteriori/plausible value) zueinander dargestellt. Der hohe Wert bei der Dimension Identifizieren unterstützt, dass das Identifizieren einen übergeordneten Faktor darstellt, der die Personen hinreichend diskriminiert. Entsprechend ergeben sich niedrigere Werte auf den zusätzlichen Dimensionen, die weitere Merkmalsbereiche erfassen. Auffällig ist allerdings, dass die beiden Werte für die Dimensionen Realisieren und Beschreiben und Begründen sehr niedrig sind. Dies könnte ein Hinweis darauf sein, dass die gebildeten Skalen und damit das Messmodell problematisch sind. ${ }^{8} \mathrm{Da}$ es sich hier um eine Reanalyse handelt, berichten wir die Ergebnisse dennoch. Diese sollten allerdings mit Vorsicht interpretiert werden und in weiterführenden Untersuchungen sollte die Eignung der EAP/PV Reliabilität zur Skalenbeurteilung bei multidimensionalen Modellen genauer betrachtet werden (Tab. 4).

Ein Vergleich der verschiedenen Modelle bezüglich der dimensionalen Struktur der Handlungen ergab, dass das 3-dimensionale Modell den niedrigsten und damit besten adj. BIC-Wert (Raftery 1995) aufweist (s. Tab. 5). Es fällt allerdings auf, dass der Abstand zum 4-dimensionalen Modell sehr gering ist. Dies könnte ein Hinweis darauf sein, dass zwar im vorliegenden Datensatz die beiden Dimensionen Beschreiben und Begründen nicht hinreichend differenzierbar sind, in einem stärker variierenden Itempool (mit mehr Items zu den beiden Dimensionen) eine Trennung der beiden Dimensionen aber möglich würde, was dem dahinter stehenden theoretischen Modell entsprechen würde. Aus den vorliegenden Ergebnissen lässt

\footnotetext{
${ }^{8}$ Anmerkung: Bei jeweils unidimensionaler Betrachtung (ohne Mehrfachladungen) resultieren höhere Varianzverhältnisse - Realisieren: 0,746; Beschreiben und Begründen: 0,702.
} 
sich bereits schlussfolgern, dass ein mehrdimensionales Modell die Daten besser beschreibt als ein 1-dimensionales Modell und dass die beiden Handlungen Identifizieren und Realisieren in jedem Fall getrennt werden können.

\subsection{Analyse der Itemschwierigkeiten}

Zusätzlich zur Überprüfung der dimensionalen Struktur der Handlungen wird im Folgenden der Frage nachgegangen, ob sich die vermutete Schwierigkeitsabstufung zwischen den beiden Handlungen Identifizieren und Realisieren in den empirischen Itemschwierigkeiten wiederspiegelt. An dieser Stelle soll betont werden, dass diese zusätzliche Analyse eher einen explorativen Charakter hat und lediglich erste Hinweise - auf Basis des vorhandenen Itempools - liefern kann. Eine detaillierte Analyse einer solchen Schwierigkeitsabstufung müsste dann in einer Folgestudie mit einem eigens dafür konzipierten Itempool erfolgen.

Die theoretische Konzeptualisierung der Handlungen legt nahe, dass sich das Realisieren auf einem höheren Schwierigkeitsniveau befindet als das Identifizieren. Dieses Ergebnis stützt die im theoretischen Modell verankerte Annahme, dass zum Lösen von Items mit Realisierungshandlung immer auch Identifizierungshandlungen erforderlich sind. Es kommt jedoch eine zusätzliche Anforderung hinzu. Bei einem Darstellungswechsel bedeutet dies, dass beim Realisieren zusätzlich die Zieldarstellungsform konstruiert werden muss.

Um eine solche Schwierigkeitsabstufung anhand der empirischen Itemschwierigkeiten betrachten zu können, müssen zunächst diejenigen Items identifiziert werden, die die gleichen mathematischen Inhalte abfragen. Dadurch soll ausgeschlossen werden, dass andere Faktoren als die integrierten Schülerhandlungen die empirischen Itemschwierigkeiten beeinflussen. Es ist beispielsweise denkbar, dass den Schülerinnen und Schülern Items zu linearen Funktionen leichter fallen als Items zu quadratischen Funktionen, da bei linearen Funktionen weniger Parameter enthalten sind. Aus diesem Grund findet zur Analyse der empirischen Itemschwierigkeit eine Beschränkung auf lineare Funktionen statt. Für diesen Gegenstandsbereich stehen mehr Items zur Verfügung. Darüber hinaus ist es möglich, dass den Lernenden bestimmte Darstellungswechsel leichter fallen als andere. Zum Beispiel ist es denkbar, dass Darstellungswechsel mit situativer Beschreibung für die Lernenden schwieriger sind, da dort zusätzlich eine Übersetzung zwischen Mathematik und „Welt“ stattfindet, im Gegensatz zu einem rein innermathematischen Darstellungswechsel wie zum Beispiel zwischen dem Darstellungswechsel zwischen Graph und Gleichung. Aus diesem Grund werden die Items zu linearen Funktionen nach Darstellungswechsel unterschieden. Der Darstellungswechsel zwischen Graph und situativer Beschreibung (GS) wurde dabei nicht berücksichtigt, da bei diesem Darstellungswechsel keine Realisieren-Items eingesetzt wurden. Für den Darstellungswechsel zwischen Graph und Gleichung (GA) findet sich ein Beispielitem zum Identifizieren in Abb. 5. Ein vergleichbares Beispielitem zum Realisieren ist in Abb. 7 dargestellt.

Die Werte für die empirischen Itemschwierigkeiten wurden aus Conquest entnommen (auf Basis des 3-dimensionalen Modells) und sind graphisch in Abb. 8 dargestellt. Bei den Darstellungswechseln graphisch-algebraisch (GA), graphischnumerisch (GN) und numerisch-algebraisch (NA) lässt sich eine deutliche Schwierig- 
Gegeben ist der folgende Graph:

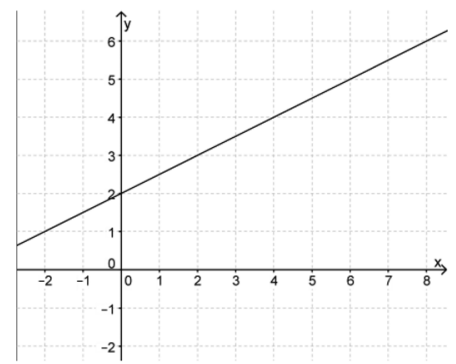

Entscheide, welche der folgenden Gleichungen zum Graphen der Funktion $\mathrm{f}$ passt und kreuze diese an.

$$
\begin{aligned}
& \square y=2 x+0,5 \\
& \square y=2 x+2 \\
& \square y=0,5 x+2 \\
& \square y=-0,5 x+2
\end{aligned}
$$

Abb. 5 Beispielitem zum Identifizieren bei linearen Funktionen

Tab. 4 Latente Korrelationen (unterhalb der Diagonalen) und EAP/PV Reliabilitäten auf den Diagonalen

\begin{tabular}{llll}
\hline & Identifizieren & Realisieren & Beschreiben und Begründen \\
\hline Identifizieren & 0,709 & & \\
Realisieren & 0,184 & 0,361 & \\
Beschreiben und Begründen & $-0,244$ & 0,428 & 0,200 \\
\hline
\end{tabular}

keitsabstufung erkennen. Einzige Ausnahme bildet der Darstellungswechsel situativ-algebraisch. Bei einer genaueren Analyse der Items in diesem Bereich fällt Folgendes auf: Bei zwei Identifizieren-Items wurde die Richtung des Darstellungswechsels, d. h. die Ausgangs- und Zieldarstellungsform vertauscht. Statt der Angabe von einer situativen Beschreibung und vier Gleichungen - wie bei allen anderen Items - sind hier eine Gleichung und vier situative Beschreibungen gegeben und die passende Situation muss ausgewählt werden. Diese Items weisen deutlich höhere Itemschwierigkeiten auf als die anderen Identifizieren-Items. In der Abb. 8 handelt es sich um die beiden grau-gefärbten Punkte. Hier scheint demnach die Richtung des Darstellungswechsels ein zusätzlicher Faktor für die Itemschwierigkeit darzustellen. Ohne Berücksichtigung dieser beiden Items findet sich auch bei diesem Darstellungswechsel die Schwierigkeitsabstufung zwischen Identifizieren- und Realisieren-Items wieder.

\section{Diskussion und Ausblick}

Die empirische Analyse des aus dem tätigkeitstheoretischen Ansatz für Schülerhandlungen resultierenden Messmodells stützt die Hypothese, dass die betrachteten Schülerhandlungen bei den untersuchten Darstellungswechseln funktionaler 


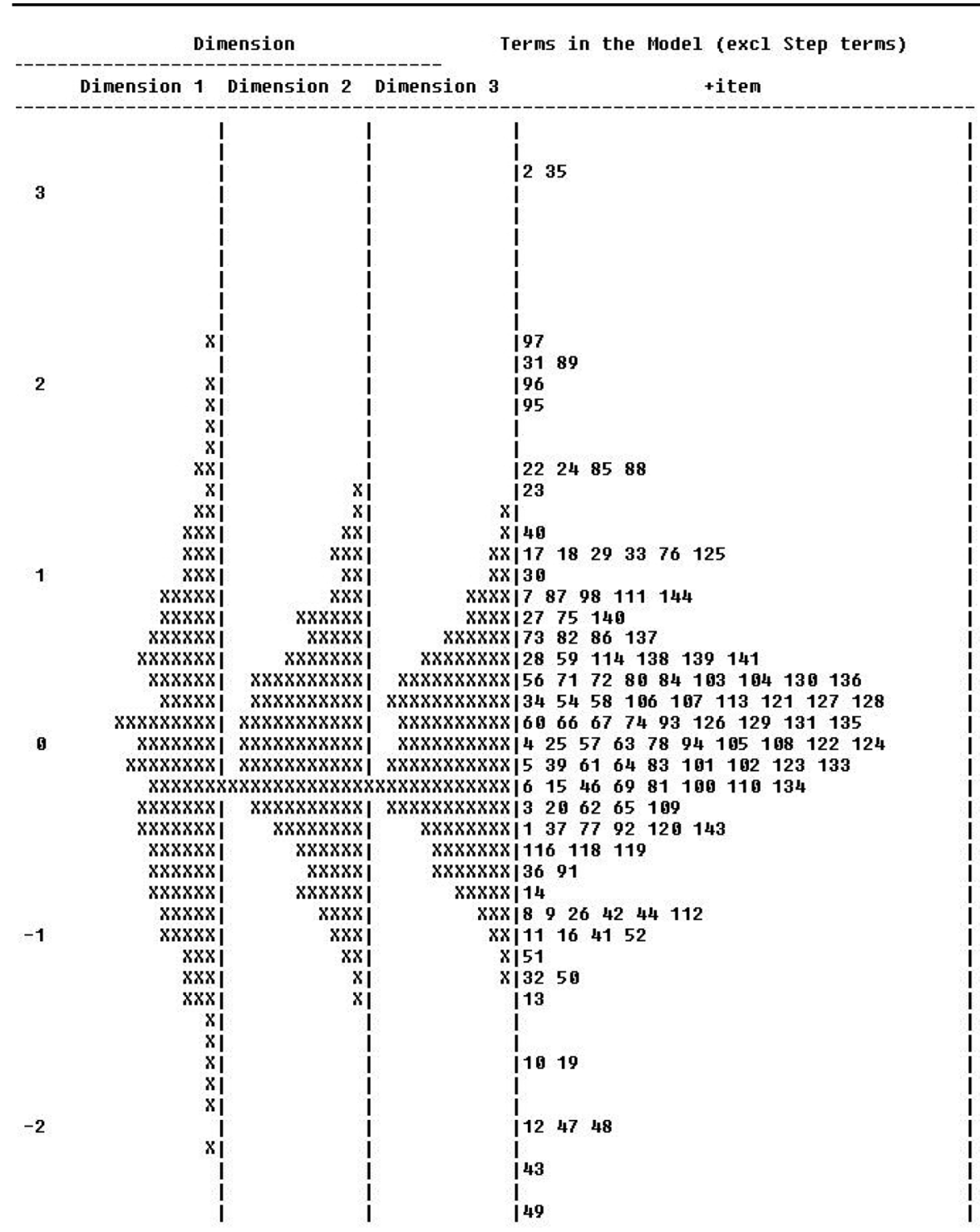

Abb. 6 Wright Map mit Personenfähigkeiten (1. bis 3. Spalte) und Itemschwierigkeiten (4. Spalte) auf einer Logit-Skala (aus CONQUEST)

Zusammenhänge kein eindimensionales Konstrukt darstellen. Aufgrund des Modellvergleichs erscheint eine mehrdimensionale Struktur naheliegend. Sowohl das 1-dimensionale als auch das 2-dimensionale Modell zeigen einen deutlich schlechteren Modellfit, da die informationstheoretischen Maße einen höheren Wert aufweisen. Offen bleibt in diesem Zusammenhang, wodurch die niedrigen Reliabilitäten der Dimensionen Realisieren und Beschreiben und Begründen zustande kommen. Eine weitere offene Frage besteht aufgrund der Modellierung des Identifizierens als eine Art ,g-Faktor“. Da im 3-dimensionalen Modell alle Items auf die Dimension Identi- 
Tab. 5 Informationskriterien zum Modellvergleich

\begin{tabular}{llll}
\hline Modell & AIC & BIC & Adj. BIC \\
\hline 1D & 19938.997 & 20528.938 & 20109.844 \\
2D - IR & 19941.860 & 20540.739 & 20115.295 \\
2D - I & 19903.920 & 20502.799 & 20077.355 \\
3D & $\mathbf{1 9 8 8 1 . 3 3 2}$ & $\mathbf{2 0 4 9 3 . 6 2 0}$ & $\mathbf{2 0 0 5 8 . 6 5 0}$ \\
4D & 19881.516 & 20511.681 & 20064.012 \\
\hline
\end{tabular}

Als wesentliches Kriterium für die Beurteilung der Modellpassung wurde hier der adjustierte BIC herangezogen. Der Vollständigkeit halber werden auch die Werte des AIC und BIC berichtet.

AIC Akaike Information Criterion, BIC Bayesian Information Criterion, Adj. BIC Adjusted Information Criterion.

fizieren laden, sind hier prinzipiell alle Aspekte enthalten, die bei allen Items für die korrekte Lösung relevant sind. Dies könnte auch Aspekte umfassen, die die Elementarhandlung des Identifizierens aus theoretischer Perspektive nicht enthält. Für eine Verifizierung der mehrdimensionalen Struktur erscheint deswegen in einer Anschlussstudie ein eigens für diese Analyse konstruierter Itempool notwendig.

Der bisherige Modellvergleich im Rahmen der Reanalyse legt nahe, dass sich die beiden Elementarhandlungen Identifizieren und Realisieren dimensional trennen lassen. Das Identifizieren wesentlicher Eigenschaften und Werte innerhalb einer/mehrerer Darstellungsformen ist dabei grundlegend. Es ist grundsätzlich in allen Aufgabenbearbeitungen notwendig. Demnach kommt dem Identifizieren eine besondere Bedeutung zu. Das Realisieren setzt ein vorheriges Identifizieren voraus, enthält jedoch zusätzliche Anforderungen, da die Zieldarstellung konstruiert werden muss. Demnach ist es von einer reinen Identifizierungshandlung zu unterscheiden. Die Grundhandlungen Beschreiben und Begründen unterscheiden sich in ihrer Anforderungsstruktur sowohl vom Identifizieren als auch vom Realisieren, was sich unter anderem durch die in den jeweiligen Items geforderte Verbalisierung erklären lässt.

Die Reanalyse stützt demnach die aus der theoretischen Konzeptualisierung folgende Hypothese, dass die beiden Handlungen Identifizieren und Realisieren für die Handlungen Beschreiben und Begründen grundlegend $\mathrm{zu}$ sein scheinen. Wir sprechen deshalb von einem hierarchischen Handlungskonzept. Identifizieren und Realisieren nehmen eine besondere Stellung ein, die bereits in den theoretischen Betrachtungen durch die Einstufung als Elementarhandlungen betont wurde und sich auch in den Beschreibungen der Grundhandlungen wiederspiegelt. Es liegt die Vermutung nahe, dass dies aufgrund der Modellkonstruktion nicht nur für das Beschreiben und Begründen gilt, sondern sich auch für andere Grundhandlungen bestätigen wird.

Die ähnlichen Ergebnisse in den Bereichen der Bruchrechnung (Dreher et al. 2014) und zu den funktionalen Zusammenhängen in unserer Studie stützen die eingangs formulierte Vermutung, dass sich die Trennung der Handlungen auf andere Inhaltsbereiche übertragen lässt, zumindest im Kontext von Darstellungswechseln. $\mathrm{Ob}$ dies wie theoretisch angenommen auch für andere Bereiche gilt, muss noch untersucht werden. 
Abb. 7 Beispielitem zum Realisieren bei linearen Funktionen
Gegeben ist folgender Graph:

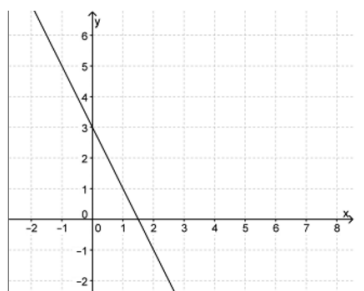

Stelle passend zum Graphen der Funktion g eine Gleichung auf.

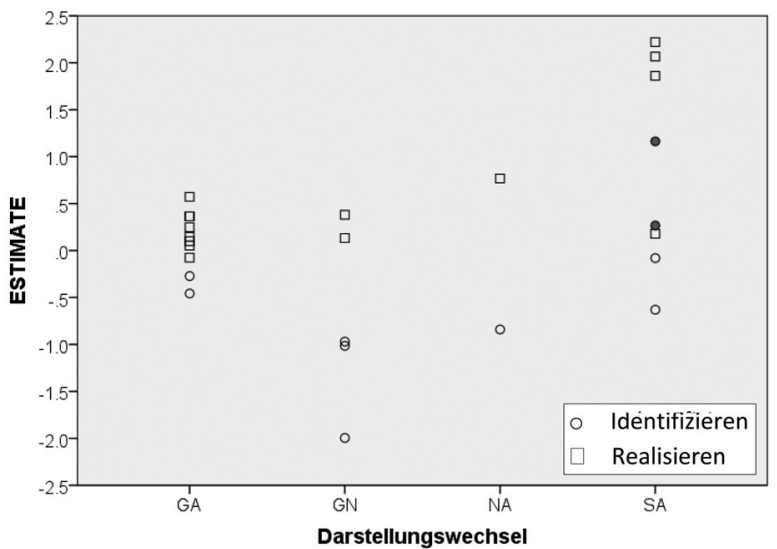

Abb. 8 Empirische Itemschwierigkeiten der vergleichbaren Identifizieren(GAI) und Realisieren-Items (GAR) zum Darstellungswechsel Graph-Gleichung bei linearen Funktionen

Inwiefern sich die beiden Handlungen Beschreiben und Begründen auch empirisch trennen lassen, ist mit dem hier betrachteten Datensatz nicht abschließend klärbar. Da das 3-dimensionale Modell (1. Identifizieren, 2. Realisieren, 3. Beschreiben und Begründen) in Abgrenzung zum 4-dimensionalen Modell den besten Modellfit aufweist, liegt die Vermutung nahe, dass die Verbalisierung, die dem Beschreiben und Begründen gemeinsam ist, eine wesentliche Komponente darstellt. Eventuell ist dennoch eine differenziertere empirische Analyse möglich, wenn ein größerer Itempool und eine größere Stichprobe zur Verfügung stehen.

Bezüglich des Potenzials unseres Ansatzes zur Beschreibung von Kompetenzstufen können aus den Ergebnissen verschiedene Schlussfolgerungen abgeleitet werden (die jedoch zunächst auf den hier betrachteten Inhaltsbereich beschränkt sind). So hat sich durch das bestätigte mehrdimensionale Modell mit Mehrfachladungen gezeigt, dass es einerseits sehr elementare Handlungen gibt, wie das Identifizieren, das in allen Aufgaben gefordert ist. Gleichzeitig konnten andere Handlungen wie das Realisieren davon abgegrenzt werden, da diese zusätzliche Anforderungen beschreiben.

Eine besondere Herausforderung bei weiterführenden Analysen in diesem Bereich besteht in der Itementwicklung. Bei den Dimensionen Realisieren und Beschreiben und Begründen muss berücksichtigt werden, dass diese nicht einzeln $-\mathrm{d}$. h. ohne Identifizieren - abbildbar sind. Es muss demnach in jedem Fall gesondert betrachtet werden, welche zusätzlichen Anforderungen bleiben, wenn die Anforderung an das Identifizieren herausgenommen wird. Diese zusätzlichen Anforderungen müssen reliabel messbar sein, ohne dass Items vorhanden sind, die ausschließlich diese 
Anforderungen messen. Die Analyse der empirischen Itemschwierigkeiten gibt ähnlich wie bei Dreher et al. (2014) erste Hinweise darauf, dass bei den beiden Handlungen Identifizieren und Realisieren eine Schwierigkeitsabstufung vorliegt. Dies unterstützt die Hypothese, dass sich die Handlungen Identifizieren und Realisieren auch zur Modellierung von Kompetenzstufen eignen. Für eine genaue Klärung und theoretische Begründung bis hin zu empirischer Prüfung von Schwierigkeitsabstufungen ist eine Folgestudie mit einem auf diese Fragestellung zugeschnittenen Itempool notwendig. Offen ist in diesem Zusammenhang die Frage, ob solche Stufungen psychometrisch mit einem Modell mit Mehrfachladungen vereinbar sind und wie sich diese unter Berücksichtigung mehrerer Dimensionen abbilden lassen.

Mit der gewählten tätigkeitstheoretischen Perspektive erscheint auch eine Modellierung von Kompetenzentwicklungen innerhalb des Lernprozesses denkbar. Eine solche Betrachtung ist durch den tätigkeitstheoretischen Hintergrund bereits theoretisch verankert, denn es ist eine der zentralen Fragen der Tätigkeitstheorie, welche Ausprägungen die Aneignung von Lerninhalten haben kann. Wie eine Aneignung mathematischen Wissens und Könnens in Abhängigkeit der Ausführung verschiedener Lernhandlungen erfolgt, darüber lässt sich jedoch zum jetzigen Zeitpunkt nur wenig aussagen. Hier sind sicherlich weiterführende Analysen notwendig, indem für die Handlungen typische Aspekte herausgearbeitet werden und die Kennzeichnung ihrer Beziehungen zu anderen Handlungen erfolgt. Durch eine solche Betrachtung wird womöglich auch deutlicher werden, wie sich Lernhandlungen auf der einen Seite und Handlungen innerhalb von Leistungssituationen auf der anderen Seite besser voneinander abgrenzen lassen.

Insgesamt soll an dieser Stelle noch einmal betont werden, dass es sich bei den vorliegenden Ergebnissen um eine Reanalyse handelt, die dazu dienen sollte, erste Hinweise für empirische Evidenzen der beschriebenen theoretischen Modellierung von Schülerhandlungen zu liefern. Für eine ausführliche Analyse in diesem Bereich bedarf es eines eigens für diese Zielstellung konstruierten Itempools.

In einem nächsten Schritt wäre es denkbar, die Kompetenzmodellierungen zu den Inhalten und den Handlungen miteinander zu verknüpfen (dazu bedarf es allerdings wiederum eines deutlich größeren Itempools). In diesem Zusammenhang könnte zusätzlich das Beschreiben und Begründen weiter ausdifferenziert werden, indem der Itemsatz eine stärkere Anforderungsvariation dieser beiden Handlungen beinhaltet. Dabei könnten dann auch die statistischen Kennwerte noch einmal detaillierter überprüft werden - auch im Hinblick auf die hier vorgefundenen problematischen Reliabilitätswerte bei einer Modellierung mit Mehrfachladungen. In diesem Bereich ist sicherlich auch noch weitere Grundlagenforschung zur Anwendung und zu Möglichkeiten und Grenzen der gewählten psychometrischen Modelle notwendig.

Entscheidend ist jedoch, dass eine strukturelle theoretische Modellierung von im jeweiligen Gegenstandsbereich relevanten Handlungsdimensionen auf einem gemeinsamen begrifflichen Hintergrund vorgenommen wird mit möglichst klarer Abgrenzung voneinander, damit dafür geeignete Messmodelle generiert werden können.

Um die theoretisch begründbare Annahme einer gegenstandsunabhängigen Unterscheidbarkeit zwischen Identifizieren und Realisieren einerseits und den Grundhandlungen Beschreiben und Begründen andererseits auf eine breitere empirische 
Verallgemeinerungsbasis zu legen, wären noch weitere Operationalisierungen in anderen Gegenstandsbereichen wünschenswert. Des Weiteren wäre es dann auch denkbar, weitere Grundhandlungen zu integrieren und deren Anforderungsstruktur aufzuklären.

Open Access Dieser Artikel unterliegt den Bedingungen der Creative Commons Attribution License. Dadurch sind die Nutzung, Verteilung und Reproduktion erlaubt, sofern der/die Originalautor/en und die Quelle angegeben sind.

\section{Literatur}

Adams, R. \& Wu, M. (2010). Differential Item Functioning. Conquest Tutorial 6. https://www.acer.edu.au/ conquest/notes-tutorials. Gesehen am: 2. Dec. 2015.

Ainsworth, S. E., Bibby, P. A., \& Wood, D. J. (2002). Examining the effects of different multiple representational systems in learning primary mathematics. Journal of the Learning Sciences, 11(1), 25-62.

Baranowski, T., Allen, D., Mâsse, L., \& Wilson, M. (2006). Does participation in an intervention affect responses on self-report questionnaires? Health Education Research, 21(1), 98-109.

Bayrhuber, M., Leuders, T., Bruder, R., \& Wirtz, M. (2010). Repräsentationswechsel beim Umgang mit Funktionen - Identifikation von Kompetenzprofilen auf der Basis eines Kompetenzstrukturmodells. In E. Klieme, D. Leutner, \& M. Kenk (Hrsg.), Kompetenzmodellierung. Zwischenbilanz des DFGSchwerpunktprogramms und Perspektiven des Forschungsansatzes, Zeitschrift Pädagogik, Beiheft 56 (S. 28-39). Weinheim: Beltz.

Biehler, R., \& Leuders, T. (2014). Kompetenzmodellierungen für den Mathematikunterricht - Eine Zwischenbilanz aus Sicht der Mathematikdidaktik. Journal für Mathematik-Didaktik, 35(1), 1-5.

Biggs, J., \& Tang, C. (2007). Teaching for quality learning at university (3rd Aufl.). Buckingham: Society for Research into Higher Education and Open University Press.

Bruder, R., \& Brückner, A. (1989). Zur Beschreibung von Schülertätigkeiten im Mathematikunterricht ein allgemeiner Ansatz. Pädagogische Forschung, 30(6), 72-82.

Bruder, R., \& Pinkernell, G. (2011). Die richtigen Argumente finden. Mathematik lehren, H. 168, 2-7

Dreher, A., Kuntze, S., \& Winkel, K. (2014). Empirical study of a competence structure model regarding conversions of representations - The case of fractions. In P. Liljedahl, S. Oesterle, C. Nicol, \& D. Allan (Hrsg.), Proceedings of the 38th Conference of the International Group for the Psychology of Mathematics Education and the 36th Conference of the North American Chapter of the Psychology of Mathematics Education (Bd. 4, S. 338-345). Vancouver: PME.

Giest, H., \& Lompscher, J. (2006). Lerntätigkeit-Lernen aus kultur-historischer Perspektive. Ein Beitrag zur Entwicklung einer neuen Lernkultur im Unterricht. Berlin: Lehmanns Media - LOB.de.

Gonzalez, E., \& Rutkowski, L. (2010). Principles of multiple matrix booklet designs and parameter recovery in largescale Assessments. In M. von Davier \& D. Hastedt (Hrsg.), IERI monograph series. Issues and methodologies in large-scale assessments (Bd. 3, S. 125-156). Hamburg: IERI.

Hartig, J., \& Höhler, J. (2008). Representation of competencies in multidimensional IRT models with within-item and between-item multidimensionality. Zeitschrift für Psychologie/Journal of Psychology, 216(2), 89-101.

Hartig, J., \& Höhler, J. (2010). Modellierung von Kompetenzen mit mehrdimensionalen IRTModellen. In E. Klieme, D. Leutner \& M. Kenk (Hrsg.), Kompetenzmodellierung: Zwischenbilanz des DFGSchwerpunktprogramms und Perspektiven des Forschungsansatzes (56. Beiheft der Zeitschrift für Pädagogik, S. 189-198). Weinheim \& Basel: Beltz.

Heinze, A. (2010). Mathematische Kompetenz modellieren und diagnostizieren: Eine Diskussion der Forschungsprojekte des DFG-Schwerpunktprogramms „Kompetenzmodelle“ aus mathematikdidaktischer Sicht. In E. Klieme, D. Leutner, \& M. Kenk (Hrsg.), Kompetenzmodellierung. Zwischenbilanz des DFG-Schwerpunktprogramms und Perspektiven des Forschungsansatzes, Zeitschrift für Pädagogik, Beiheft; 56 (S. 86-91). Weinheim: Beltz.

Klieme, E. (2004). Was sind Kompetenzen und wie lassen sie sich messen? Pädagogik, 6, 10-13. 
Klieme, E., \& Leutner, D. (2006). Kompetenzmodelle zur Erfassung individueller Lernergebnisse und zur Bilanzierung von Bildungsprozessen. Beschreibung eines neu eingerichteten Schwerpunktprogramms der DFG. Zeitschrift für Pädagogik, 52(6), 876-903.

Klieme, E., et al. (2007). Zur Entwicklung nationaler Bildungsstandards. In Bundesministerium für Bildung und Forschung Hrsg, Bildungsforschung (Bd. 1). http://www.bmbf.de/pub/zur_entwicklung_nationaler_bildungsstandards.pdf. Gesehen am: 11. Sept. 2013.

Klieme, E., Hartig, J., \& Rauch, D. (2008). The concept of competence in educational contexts. In J. Hartig, E. Klieme, \& D. Leutner (Hrsg.), Assessment of competencies in educational contexts (S. 3-22). Göttingen: Hogrefe.

Koeppen, K., Hartig, J., Klieme, E., \& Leutner, D. (2008). Current Issues in Competence Modeling and Assessment. Zeitschrift für Psychologie, 216(2), 61-73.

Kossakowski, A., \& Lompscher, J. (1988). Zum Tätigkeitskonzept in der Psychologie. Päd. Forschung, 29(1), 28-50.

Leinhardt, G., Zaslavsky, O., \& Stein, M. K. (1990). Functions, Graphs, and Graphing: Tasks, Learning, and Teaching. Review of Educational Research, 60(1), 1-64.

Leont'ev, A. N. (1977). Tätigkeit, Bewusstsein, Persönlichkeit. Stuttgart: Klett.

Lompscher, J. (1972). Theoretische und experimentelle Untersuchungen zur Entwicklung geistiger Fähigkeiten. Berlin: Volk und Wissen.

Lompscher, J. (1990). Konzept(e) der Lerntätigkeit. Überarbeitetes und ins Deutsche übersetztes Einleitungsreferat zum gleichnamigen Symposium auf dem 2. Internationalen Kongress zur Tätigkeitstheorie. Lahti (Finnland), Mai 1990. https://publishup.uni-potsdam.de/opus4-ubp/files/391/ KONZEPT.pdf.

Lurija, A. R. (1992). Die moderne Psychologie und der dialektische Materialismus. In W. Jantzen \& M. Holodynskij. (Hrsg.), Studien zur Tätigkeitstheorie VII-A. R. Lurija heute (S. 5-26). Bremen: Luria Gesellschaft e. V.

Moosbrugger, H., \& Kelava, A. (Hrsg.). (2007). Testtheorie und Fragebogenkonstruktion. Heidelberg: Springer.

Muthén, L. K., \& Muthén, B. O. (1998-2010). Mplus user's guide. 6. Aufl. Los Angeles: Muthén \& Muthén.

Nitsch, R., Fredebohm, A., Bruder, R., Kelava, T., Naccarella, D., Leuders, T., \& Wirtz, M. (2015). Students' competencies in working with functions in secondary mathematics education - Empirical examination of a competence structure model. International Journal of Science and Mathematics Education, 13(3), 657-682.

Raftery, A. E. (1995). Bayesian model selection in social research. Sociological Methodology, 25, 111-163.

Rasch, G. (1960/1980). Probabilistic models for some intelligence and attainment tests. (Copenhagen, Danish Institute for Educational Research), expanded edition (1980) with foreword and afterword by B.D. Wright. Chicago: The University of Chicago Press.

Rupp, A. A., \& Mislevy, R. J. (2007). Cognitive foundations of structured item response theory models. In J. Leighton \& M. Gierl (Hrsg.), Cognitive diagnostic assessment in education: Theory and applications (S. 205-241). Cambridge: Cambridge University Press.

Schecker, H., \& Parchmann, I. (2006). Modellierung naturwissenschaftlicher Kompetenz. Zeitschrift für Didaktik der Naturwissenschaften, 12, 45-66.

Sclove, L. (1987). Application of model-selection criteria to some problems in multivariate analysis. Psychometrika, 52, 333-343.

Siller, H.-S., Bruder, R., Hascher, T., \& Linnemann, T. (2014). Abschlussbericht zum Projekt „,Kompetenzmodellierung für die schriftliche MATURA in Mathematik". bifie Wien (im Druck).

Steinhöfel, W., Reichold, K., \& Frenzel, L. (1985). Zur Gestaltung typischer Unterrichtssituationen im Mathematikunterricht. Lehrmaterial zur Ausbildung von Diplomlehrern Mathematik, Potsdam.

Swan, M. (1985). The language of functions and graphs. Nottingham: Shell Centre for Mathematical Education.

Vandebrouck, F., Monaghan, J., \& Lagrange, J.-B. (2013). Activity theoretical approaches to mathematics classroom practices with the use of technology. In A. M. Lindmeier \& A. Heinze (Hrsg.), Proceedings of the 37th Conference of the International Group for the Psychology of Mathematics Education (1, S. 181-210). Kiel: PME.

Vollrath, H.-J. (1989). Funktionales Denken. Journal für Mathematik-Didaktik, 10, 3-37.

Vom Hofe, R. (1995). Grundvorstellungen mathematischer Inhalte. Heidelberg: Spektrum Akademischer Verlag. 
Vygotsky, L. S. (1978). Mind in society. The development of higher psychological processes. Cambridge: Harvard University Press.

Walker, C. M., \& Beretvas, S. N. (2003). Comparing multidimensional and unidimensional proficiency classifications: Multidimensional IRT as a diagnostic aid. Journal of Educational Measurement, 40(3), $255-275$.

Weinert, F. E. (2001). Vergleichende Leistungsmessung in Schulen - eine umstrittene Selbstverständlichkeit. In F. E. Weinert (Hrsg.), Leistungsmessungen in Schulen (S. 17-31). Weinheim: Beltz.

Wilson, M., De Boeck, P., \& Carstensen, C. H. (2008). Explanatory item response models: A brief introduction. In J. Hartig, E. Klieme \& D. Leutner (Hrsg.), Assessment of competencies in educational contexts (S. 91-120). Göttingen: Hogrefe.

Wu, M. L., Adams, R. J., Wilson, M., \& Haldane, S. (2007). ConQuest Version 2.0. St. Paul: Assessment Systems Corporation. 